TRANSACTIONS OF THE

AMERICAN MATHEMATICAL SOCIETY

Volume 359, Number 3, March 2007, Pages 1351-1374

S 0002-9947(06)04109-2

Article electronically published on September 19, 2006

\title{
CM POINTS ON PRODUCTS OF DRINFELD MODULAR CURVES
}

\author{
FLORIAN BREUER
}

\begin{abstract}
Let $X$ be a product of Drinfeld modular curves over a general base ring $A$ of odd characteristic. We classify those subvarieties of $X$ which contain a Zariski-dense subset of CM points. This is an analogue of the AndréOort conjecture. As an application, we construct non-trivial families of higher Heegner points on modular elliptic curves over global function fields.
\end{abstract}

\section{INTRODUCTION}

1.1. Basic notations. The following notations will be used throughout this paper. Let $\mathbb{F}_{q}$ denote the finite field with $q$ elements, where $q$ is a power of the odd prime $p$. Let $k$ be a global function field with field of constants $\mathbb{F}_{q}$. Fix a place $\infty$ of $k$, and denote by $k_{\infty}$ the completion of $k$ at $\infty$, and by $\mathbb{C}_{\infty}=\hat{\bar{k}}_{\infty}$ the completion of an algebraic closure of $k_{\infty}$. Let $A=\{x \in k \mid x$ is regular outside $\infty\}$; it is a Dedekind domain of finite class number. We denote by $|\cdot|$ the absolute value corresponding to $\infty$, and note that for $a \in A$, we have $|a|=q^{\operatorname{deg}(a)}=|A /\langle a\rangle|$.

Unless stated otherwise, a Drinfeld module always means a rank 2 Drinfeld $A$ module defined over $\mathbb{C}_{\infty}$ of generic characteristic. See [9] and [11] for an overview of Drinfeld modules.

We denote by $\hat{A}=\lim A / \mathfrak{n}$ the profinite completion of $A$, and by $\mathbb{A}_{f}=\hat{A} \otimes_{A}$ $k$ the ring of finite adèles of $k$. Let $\mathcal{K}$ be a subgroup of finite index (i.e. an open subgroup) of $\mathrm{GL}_{2}(\hat{A})$. Then we denote by $M_{A}^{2}(\mathcal{K})$ the coarse moduli scheme parameterizing rank 2 Drinfeld $A$-modules with level $\mathcal{K}$-structure; it is an affine curve over $\operatorname{Spec}(A)$, which is not in general irreducible. A Drinfeld modular curve over $\mathbb{C}_{\infty}$ is an irreducible component of some $M_{A}^{2}(\mathcal{K})_{\mathbb{C}_{\infty}}=M_{A}^{2}(\mathcal{K}) \times_{A} \mathbb{C}_{\infty}$. If $\mathcal{K}=\mathrm{GL}_{2}(\hat{A})$ we will just write $M_{A}^{2}=M_{A}^{2}\left(\mathrm{GL}_{2}(\hat{A})\right)$, which is the coarse moduli space of Drinfeld modules without level structure.

Let $\Omega:=\mathbb{P}^{1}\left(\mathbb{C}_{\infty}\right)-\mathbb{P}^{1}\left(k_{\infty}\right)$ denote the Drinfeld upper half-plane, which has a rigid analytic structure. The group $\mathrm{GL}_{2}\left(k_{\infty}\right)$ acts on $\Omega$ via fractional linear transformations. Let $X$ be a Drinfeld modular curve. Then it is known that, as rigid analytic varieties, we have

$$
X\left(\mathbb{C}_{\infty}\right)^{\text {an }} \cong \Gamma \backslash \Omega
$$

Received by the editors September 20, 2004 and, in revised form, March 1, 2005.

2000 Mathematics Subject Classification. Primary 11G09; Secondary 14G35.

Key words and phrases. Drinfeld modular curves, CM points, André-Oort conjecture, Heegner points.

(C)2006 American Mathematical Society Reverts to public domain 28 years from publication 
for some arithmetic subgroup $\Gamma \subset \mathrm{GL}_{2}(k)$. We denote by $[\omega] \in X\left(\mathbb{C}_{\infty}\right)$ the point corresponding to $\omega \in \Omega$.

1.2. Main results. Let $X=X_{1} \times \cdots \times X_{n}$ be a product of Drinfeld modular curves. A point $x=\left(x_{1}, \ldots, x_{n}\right) \in X\left(\mathbb{C}_{\infty}\right)$ is called a $C M$ point if each $x_{i}$ corresponds to a Drinfeld module with complex multiplication. These $x_{i}$ in turn correspond via (1.1) to points $\omega_{i} \in \Omega$ which, viewed as elements of $\mathbb{C}_{\infty}$, are quadratic over $k$, i.e. $\left[k\left(\omega_{i}\right): k\right]=2$.

An irreducible algebraic subcurve $Y \subset X$ is called a special subcurve if there exists a partition $\{1, \ldots, n\}=I_{0} \amalg I_{1}, I_{1} \neq \emptyset$, and elements $g_{i} \in \mathrm{GL}_{2}(k)$, for all $i \in I_{1}$, such that $Y=\{x\} \times Y^{\prime}$, where $x \in \prod_{i \in I_{0}} X_{i}\left(\mathbb{C}_{\infty}\right)$ is a $C M$ point and $Y^{\prime} \subset \prod_{i \in I_{1}} X_{i}$ is a curve such that $Y^{\prime}\left(\mathbb{C}_{\infty}\right)$ is the image of the map

$$
\Omega \longrightarrow \prod_{i \in I_{1}} X_{i}\left(\mathbb{C}_{\infty}\right) ; \quad \omega \longmapsto\left(\left[g_{i}(\omega)\right]\right)_{i \in I_{1}} .
$$

The curve $Y$ is called a pure special subcurve if $I_{0}=\emptyset$, i.e. if the projections $p_{i}: Y \rightarrow X_{i}$ are surjective for all $i=1, \ldots, n$.

An irreducible algebraic subvariety $Y \subset X$ is called a special subvariety if there exists a partition $\{1, \ldots, n\}=\coprod_{j=0}^{g} I_{j}$ such that $Y=\{x\} \times \prod_{j=1}^{g} Y_{j}$, where $x \in$ $\prod_{i \in I_{0}} X_{i}\left(\mathbb{C}_{\infty}\right)$ is a $\mathrm{CM}$ point, and each $Y_{j} \subset \prod_{i \in I_{j}} X_{i}$ is a pure special subcurve, for $j=1, \ldots, g$. We see that $\mathrm{CM}$ points are just special subvarieties of dimension zero.

The aim of this paper is to prove the following result.

Theorem 1.2. Let $X=X_{1} \times \cdots \times X_{n}$ be a product of Drinfeld modular curves. Then an irreducible algebraic subvariety $Y \subset X$ contains a Zariski-dense subset of $C M$ points if and only if $Y$ is a special subvariety.

It is easy to see that a special subvariety contains a Zariski-dense set of CM points, the hard part is to prove the converse. In the special case where $Y$ is a curve, we actually prove an effective result, namely that $Y$ is special if and only if $Y$ contains a CM point of sufficiently large $C M$ height; see Theorem 3.25

Theorem 1.2 is an analogue of the André-Oort Conjecture for products of classical modular curves; see [1] and 4]. In fact, our proof is closely modeled on Edixhoven's approach [4].

Theorem 1.2 was proved in 3 for the special case $A=\mathbb{F}_{q}[T]$. In this paper we show how to adapt the arguments of $[3$ to the case of general $A$ (but still of odd characteristic). As an application, in Section 4 we extend our previous results 2 . concerning higher Heegner points on elliptic curves over rational functions fields to the case of global function fields.

\section{Some PRELIMINARIES}

We begin by gathering some basic results which will be needed in the proof of Theorem 1.2 .

2.1. Complex multiplication and CM heights. Let $\phi$ be a Drinfeld module with complex multiplication by the ring $R$, and let $K$ be the quotient field of $R$. Then $K / k$ is a quadratic imaginary extension, which means that only one prime of $K$ lies above $\infty$, which we again call $\infty$. Denote by $\mathcal{O}_{K}$ the ring of integers of 
$K$, i.e. the integral closure of $A$ in $K$. Then $A \subset R \subset \mathcal{O}_{K}$, and $R$ is a projective $A$-module of rank 2, hence by the invariant factor theorem, $R=A+\mathfrak{f} \mathcal{O}_{K}$, for some ideal $\mathfrak{f} \subset A$, which we will call the conductor of $R$. Note that $\mathfrak{f} \mathcal{O}_{K}$ is the largest $\mathcal{O}_{K}$-ideal which is also an $R$-ideal, which is the definition of conductor usually found in the literature.

The ring $R$ is an order in $K$, and is not in general integrally closed. However, one may still view $\phi$ as a Drinfeld $R$-module of rank 1, after Hayes 10, and in fact we have $M_{R}^{1}=\operatorname{Spec}\left(\mathcal{O}_{H_{R}}\right)$, where $\mathcal{O}_{H_{R}}$ is the ring of integers of the class field $H_{R} / K$ corresponding to the class group $K^{\times} \backslash \mathbb{A}_{f, K}^{\times} / \hat{R}^{\times} \cong \operatorname{Pic}(R)$. The field $H_{R}$ is also known as the ring class field of $R$, and $H_{R} / K$ is unramified outside $\mathfrak{f O}_{K}$. Note that we only deal with the finite adèles $\mathbb{A}_{f, K}$, so that $H_{R} / K$ splits completely at $\infty$. The action of $\mathbb{A}_{f, K}^{\times}$on $\operatorname{Spec}\left(\mathcal{O}_{H_{R}}\right)$ coincides, via class field theory, with the action of $\operatorname{Gal}\left(H_{R} / K\right)$ on $M_{R}^{1}$. Hence $\phi$ is defined over $H_{R}$ and isogenies act like Galois. In particular, we have

Proposition 2.1. Let $\phi$ be a Drinfeld module with complex multiplication by $R$. Let $\mathfrak{n} \subset A$ be a non-zero ideal such that every prime factor of $\mathfrak{n}$ splits in $R$, and let $\sigma_{\mathfrak{n}}=\left(\mathfrak{n}, H_{R} / k\right) \in \operatorname{Gal}\left(H_{R} / k\right)$ be the corresponding Frobenius element. Then $\phi$ and $\phi^{\sigma_{\mathfrak{n}}}$ are linked by a cyclic isogeny of degree $\mathfrak{n}$.

Proof. As every prime factor of $\mathfrak{n}$ splits in $R$, we may choose an ideal $\mathfrak{N}$ of $R$ such that $R / \mathfrak{N} \cong A / \mathfrak{n}$. Now an isogeny of $\phi$ as a rank 1 Drinfeld $R$-module with kernel $R / \mathfrak{N}$ is also an isogeny of $\phi$ as a rank 2 Drinfeld $A$-module with kernel $A / \mathfrak{n}$. The result now follows from the above discussion.

Note that $H_{R} / k$ might not be abelian, but since all prime factors of $\mathfrak{n}$ split in $K / k$ and $H_{R} / K$ is abelian, the conjugacy class $\left(\mathfrak{n}, H_{R} / k\right)$ contains only the one element $\sigma_{\mathfrak{n}}=\left(\mathfrak{N}, H_{R} / K\right)$.

Denote $|\mathfrak{f}|=|A / \mathfrak{f}|$; then we define the $C M$ height of $\phi$ by

$$
H_{\mathrm{CM}}(\phi):=q^{g}|\mathfrak{f}|,
$$

where $g$ is the genus of $K$. Note that this definition differs from [3, Def. 3.7] by a power of $1 / 2$. If $x=\left(x_{1}, \ldots, x_{n}\right) \in M_{A}^{2}\left(\mathcal{K}_{1}\right)\left(\mathbb{C}_{\infty}\right) \times \cdots \times M_{A}^{2}\left(\mathcal{K}_{n}\right)\left(\mathbb{C}_{\infty}\right)$ is a $\mathrm{CM}$ point, then we define

$$
H_{\mathrm{CM}}(x):=\max \left(H_{\mathrm{CM}}\left(x_{1}\right), \ldots, H_{\mathrm{CM}}\left(x_{n}\right)\right) .
$$

The following result shows that $H_{\mathrm{CM}}$ is a counting function on the set of $\mathrm{CM}$ points in $M_{A}^{2}\left(\mathbb{C}_{\infty}\right)$, which justifies the terminology.

Proposition 2.3. For every $B>0$, the set

$$
\left\{\phi \in M_{A}^{2}\left(\mathbb{C}_{\infty}\right) \mid \phi \text { is } C M \text { and } H_{\mathrm{CM}}(\phi)<B\right\}
$$

is finite.

Proof. For a given $g \geq 0$ there are only finitely many global function fields $K$ with genus $g$ and field of constants contained in $\mathbb{F}_{q^{2}}$. For each such field $K$ which is quadratic over $k$, there are only finitely many orders of the form $R=A+\mathfrak{f} \mathcal{O}_{K}$ with bounded $\mathfrak{f}$. For each such $R$, there are only $|\operatorname{Pic}(R)|$ Drinfeld modules $\phi$ with $\operatorname{End}(\phi) \cong R$. 
We will need the following class-number estimate.

Proposition 2.4. Let $\phi$ be a Drinfeld module with complex multiplication by $R$. Then for every $\varepsilon>0$ there exists a computable constant $C_{\varepsilon}>0$ such that

$$
|\operatorname{Pic}(R)|>C_{\varepsilon} H_{\mathrm{CM}}(\phi)^{1-\varepsilon} \text {. }
$$

Proof. Let $K$ be the quotient field of $R$, and $g$ its genus. First, we have [3, Prop. $3.1]$

$$
\left|\operatorname{Pic}\left(\mathcal{O}_{K}\right)\right| \geq h(K) \geq \frac{(q-1)\left(q^{2 g}-2 g q^{g}+1\right)}{2 g\left(q^{g+1}-1\right)},
$$

where $h(K)$ denotes the class number of $K$. Second, the exact sequence [15, §I.12]

$$
1 \rightarrow \mathcal{O}_{K}^{\times} / R^{\times} \longrightarrow\left(\mathcal{O}_{K} / \mathfrak{f} \mathcal{O}_{K}\right)^{\times} /\left(R / \mathfrak{f} \mathcal{O}_{K}\right)^{\times} \longrightarrow \operatorname{Pic}(R) \longrightarrow \operatorname{Pic}\left(\mathcal{O}_{K}\right) \rightarrow 1
$$

leads, as in the classical case, to the pleasing expression

$$
|\operatorname{Pic}(R)|=\frac{\left|\operatorname{Pic}\left(\mathcal{O}_{K}\right)\right|}{\left[\mathcal{O}_{K}^{\times}: R^{\times}\right]} \cdot|\mathfrak{f}| \prod_{\mathfrak{p} \mid \mathfrak{f}}\left(1-\chi(\mathfrak{p})|\mathfrak{p}|^{-1}\right),
$$

where

$$
\chi(\mathfrak{p})=\left\{\begin{aligned}
1 & \text { if } \mathfrak{p} \text { splits in } K / k, \\
-1 & \text { if } \mathfrak{p} \text { is inert in } K / k, \\
0 & \text { if } \mathfrak{p} \text { is ramified in } K / k .
\end{aligned}\right.
$$

Here one uses the fact that $R / \mathfrak{f} \mathcal{O}_{K} \cong A / \mathfrak{f}$. The estimate now follows easily.

2.2. Drinfeld modular curves. Let $\mathcal{K} \subset \mathrm{GL}_{2}(\hat{A})$ be a subgroup of finite index, and recall that $M_{A}^{2}(\mathcal{K})$ denotes the coarse moduli scheme parameterizing Drinfeld modules with level- $\mathcal{K}$ structure. Identifying Drinfeld modules over $\mathbb{C}_{\infty}$ with their associated rank 2 lattices in $\mathbb{C}_{\infty}$, and parameterizing the space of such lattices adèlically, one arrives at the following analytic parametrization (see e.g. 7]):

$$
\begin{aligned}
M_{A}^{2}(\mathcal{K})\left(\mathbb{C}_{\infty}\right)^{\text {an }} & \cong \mathrm{GL}_{2}(k) \backslash \Omega \times \mathrm{GL}_{2}\left(\mathbb{A}_{f}\right) / \mathcal{K} \\
& \cong \coprod_{s \in S} \Gamma_{s} \backslash \Omega,
\end{aligned}
$$

where $S \subset \mathrm{GL}_{2}\left(\mathbb{A}_{f}\right)$ denotes a (finite) set of representatives for $\mathrm{GL}_{2}(k) \backslash \mathrm{GL}_{2}\left(\mathbb{A}_{f}\right) / \mathcal{K}$, and $\Gamma_{s}:=s \mathcal{K} s^{-1} \cap \mathrm{GL}_{2}(k)$.

The determinant map induces a bijection

$$
\text { det : } \mathrm{GL}_{2}(k) \backslash \mathrm{GL}_{2}\left(\mathbb{A}_{f}\right) / \mathcal{K} \stackrel{\sim}{\longrightarrow} k^{\times} \backslash \mathbb{A}_{f}^{\times} / \operatorname{det}(\mathcal{K}) \quad\left(\cong \operatorname{Pic}(A) \text { if } \operatorname{det}(\mathcal{K})=\hat{A}^{\times}\right) .
$$

We next describe a scheme-theoretic version of the determinant map. Let $f \in A$, and denote by $\mathcal{K}(f) \subset \mathrm{GL}_{2}(\hat{A})$ the kernel of reduction mod $f$. The Weil pairing for Drinfeld modules [13, Chap. 5] gives us a map of $A[1 / f]$-schemes

$$
M_{A}^{2}(\mathcal{K}(f)) \stackrel{w_{f}}{\longrightarrow} M_{A}^{1}(\operatorname{det}(\mathcal{K}(f)))=\operatorname{Spec}\left(\mathcal{O}_{H_{f}}\right),
$$

where $\mathcal{O}_{H_{f}}$ denotes the ring of integers of the class field $H_{f}$ of $k$ corresponding to the class group $k^{\times} \backslash \mathbb{A}_{f}^{\times} / \operatorname{det}(\mathcal{K}(f))=k^{\times} \backslash \mathbb{A}_{f}^{\times} /\left\{x \in \hat{A}^{\times} \mid x \equiv 1 \bmod f\right\}$. Under this map, the left $\mathbb{A}_{f}^{\times} \mathrm{GL}_{2}(\hat{A})$-action on $M_{A}^{2}(\mathcal{K}(f))$ corresponds to its determinant $\mathbb{A}_{f}^{\times}$-action on $\operatorname{Spec}\left(\mathcal{O}_{H_{f}}\right)$, which in turn corresponds to the $\operatorname{Gal}\left(k^{\mathrm{ab}} / k\right)$-action on both sides given by the reciprocity map. It follows that $M_{A}^{2}(\mathcal{K}(f)) \times_{A} k$ is defined over $k$, and all its $\bar{k}$-irreducible components are defined over $H_{f}$. 
Suppose now that $\mathcal{K}$ contains $\mathcal{K}(f)$ for some $f \in A$, so $\mathcal{K}$ is a congruence subgroup. Then we may divide out by the action of $\mathcal{K}$ and $\operatorname{det}(\mathcal{K})$ in the left- and right-hand sides of (2.8), respectively. As $M_{A}^{2}(\mathcal{K})=\mathcal{K} \backslash M_{A}^{2}(\mathcal{K}(f))$, we thus get a map of $A[1 / f]$-schemes

$$
M_{A}^{2}(\mathcal{K}) \stackrel{w_{\mathcal{K}}}{\longrightarrow} M_{A}^{1}(\operatorname{det}(\mathcal{K}))=\operatorname{Spec}\left(\mathcal{O}_{H_{\mathcal{K}}}\right),
$$

where this time $H_{\mathcal{K}}$ is the class field corresponding to $k^{\times} \backslash \mathbb{A}_{f}^{\times} / \operatorname{det}(\mathcal{K})$. As before, it follows that $M_{A}^{2}(\mathcal{K}) \times{ }_{A} k$ is defined over $k$, and its $\bar{k}$-irreducible components are defined over $H_{\mathcal{K}}$.

In the case $\mathcal{K}=\mathrm{GL}_{2}(\hat{A})$, recall that we use the notation $M_{A}^{2}:=M_{A}^{2}\left(\mathrm{GL}_{2}(\hat{A})\right)$. Let $H$ denote the Hilbert class field of $k$, corresponding to the class group $\operatorname{Pic}(A) \cong$ $k^{\times} \backslash \mathbb{A}_{f}^{\times} / \hat{A}^{\times}$. It is the maximal unramified abelian extension of $k$ in which $\infty$ splits completely. Then we see that the irreducible components of $M_{A, \mathbb{C}_{\infty}}^{2}$ are defined over $H$. We also see that its set of irreducible components corresponds to $\operatorname{Pic}(A)$ in such a way that the component corresponding to $[\mathfrak{a}] \in \operatorname{Pic}(A)$ parametrizes lattices isomorphic to $A \oplus \mathfrak{a}$ as projective $A$-modules.

We may choose our representatives $S$ such that $1 \in S$, in which case $\Gamma_{1}=$ $\mathrm{GL}_{2}(A)$, and one of the irreducible components of $M_{A, \mathbb{C}_{\infty}}^{2}$ thus corresponds to $\mathrm{GL}_{2}(A) \backslash \Omega$ via (2.6). We call it the main component, and denote it by $\mathbb{M}$. It is the simplest modular curve. If $A=\mathbb{F}_{q}[T]$, then in fact $\mathbb{M} \cong \mathbb{A}^{1}$ is the affine line. In general, we have seen that $\mathbb{M}$ is a smooth irreducible affine curve defined over $H$.

2.3. The curves $Y(\mathfrak{n}), Y_{0}(\mathfrak{n})$ and $Y_{2}(\mathfrak{n})$. We denote by $Z$ the center of the algebraic group $\mathrm{GL}_{2}$. Let $\mathfrak{n} \subset A$ be a non-zero ideal, and consider the following open subgroups of $\mathrm{GL}_{2}(\hat{A})$ :

$$
\begin{aligned}
\mathcal{K}(\mathfrak{n}) & :=\operatorname{ker}\left(\mathrm{GL}_{2}(\hat{A}) \rightarrow \mathrm{GL}_{2}(A / \mathfrak{n})\right), \\
\mathcal{K}_{0}(\mathfrak{n}) & :=\left\{\left(\begin{array}{ll}
a & b \\
c & d
\end{array}\right) \in \mathrm{GL}_{2}(\hat{A}) \mid c \in \mathfrak{n} \hat{A}\right\} \\
\mathcal{K}_{2}(\mathfrak{n}) & :=\left\{\gamma \in \mathrm{GL}_{2}(\hat{A}) \mid(\gamma \bmod \mathfrak{n}) \in Z(A / \mathfrak{n})\right\}
\end{aligned}
$$

These lead to coarse moduli schemes $M_{A}^{2}(\mathcal{K}(\mathfrak{n}))$ (also denoted $M_{A}^{2}(\mathfrak{n})$ in the literature), $M_{A}^{2}\left(\mathcal{K}_{0}(\mathfrak{n})\right)$ and $M_{A}^{2}\left(\mathcal{K}_{2}(\mathfrak{n})\right)$. Similar to 2.2 , we define the Drinfeld modular curves $Y(\mathfrak{n}), Y_{0}(\mathfrak{n})$ and $Y_{2}(\mathfrak{n})$ as the main components of the respective moduli spaces over $\mathbb{C}_{\infty}$.

We have isomorphisms of rigid analytic varieties

$$
Y(\mathfrak{n})\left(\mathbb{C}_{\infty}\right)^{\text {an }} \cong \Gamma(\mathfrak{n}) \backslash \Omega, \quad Y_{0}(\mathfrak{n})\left(\mathbb{C}_{\infty}\right)^{\text {an }} \cong \Gamma_{0}(\mathfrak{n}) \backslash \Omega, \quad Y_{2}(\mathfrak{n})\left(\mathbb{C}_{\infty}\right)^{\text {an }} \cong \Gamma_{2}(\mathfrak{n}) \backslash \Omega,
$$

for the arithmetic groups

$$
\begin{aligned}
\Gamma(\mathfrak{n}) & =\left\{\gamma \in \mathrm{GL}_{2}(A) \mid \gamma \equiv 1 \bmod \mathfrak{n}\right\}, \\
\Gamma_{0}(\mathfrak{n}) & =\left\{\left(\begin{array}{ll}
a & b \\
c & d
\end{array}\right) \in \mathrm{GL}_{2}(A) \mid c \in \mathfrak{n}\right\}, \\
\Gamma_{2}(\mathfrak{n}) & =\left\{\gamma \in \mathrm{GL}_{2}(A) \mid(\gamma \bmod \mathfrak{n}) \in Z(A / \mathfrak{n})\right\} .
\end{aligned}
$$

As in the classical case, $Y_{0}(\mathfrak{n})$ parametrizes pairs of Drinfeld modules linked by cyclic $\mathfrak{n}$-isogenies, but where the first Drinfeld module corresponds to a lattice isomorphic to $A^{2}$ as an $A$-module.

As $\mathcal{K}(\mathfrak{n}), \mathcal{K}_{0}(\mathfrak{n})$ and $\mathcal{K}_{2}(\mathfrak{n})$ are all congruence subgroups of $\mathrm{GL}_{2}(\hat{A})$ (pick any $0 \neq f \in \mathfrak{n})$, it follows from the Weil-pairing (2.9) that $Y_{0}(\mathfrak{n})$ is defined over $H$, 
whereas the curves $Y(\mathfrak{n})$ and $Y_{2}(\mathfrak{n})$ are defined over the class fields corresponding to

$$
\begin{array}{r}
k^{\times} \backslash \mathbb{A}_{f}^{\times} /\left\{x \in \hat{A}^{\times} \mid x \equiv 1 \bmod \mathfrak{n}\right\} \text { and } \\
k^{\times} \backslash \mathbb{A}_{f}^{\times} /\left\{x \in \hat{A}^{\times} \mid x \text { is a square } \bmod \mathfrak{n}\right\},
\end{array}
$$

respectively.

For a ring $R \supset \mathbb{F}_{q}$, and algebraic group $G$, we define

$$
G^{1}(R):=\left\{g \in G(R) \mid \operatorname{det}(g) \in \mathbb{F}_{q}^{\times}\right\} .
$$

The degree of an ideal $\mathfrak{n} \subset A$ is $\operatorname{deg}(\mathfrak{n}):=\log _{q}|\mathfrak{n}|=\log _{q}|A / \mathfrak{n}|$.

Proposition 2.10. We have covers $Y(\mathfrak{n}) \rightarrow Y_{2}(\mathfrak{n}) \rightarrow Y_{0}(\mathfrak{n}) \rightarrow \mathbb{M}$. Moreover, $Y(\mathfrak{n})$ and $Y_{2}(\mathfrak{n})$ are Galois covers of $\mathbb{M}$ with Galois groups

$$
\begin{aligned}
\operatorname{Gal}(Y(\mathfrak{n}) / \mathbb{M}) & \cong \operatorname{GL}_{2}^{1}(A / \mathfrak{n}) / Z\left(\mathbb{F}_{q}\right), \\
\operatorname{Gal}\left(Y_{2}(\mathfrak{n}) / \mathbb{M}\right) & \cong \operatorname{GL}_{2}^{1}(A / \mathfrak{n}) / Z^{1}(A / \mathfrak{n}) \cong \operatorname{PGL}_{2}^{1}(A / \mathfrak{n}) .
\end{aligned}
$$

Note that $\operatorname{PGL}_{2}^{1}(A / \mathfrak{n}) \cong \operatorname{PSL}_{2}(A / \mathfrak{n})$ if every prime factor of $\mathfrak{n}$ has even degree.

Proof. Clearly, $\Gamma(\mathfrak{n}) \subset \Gamma_{2}(\mathfrak{n}) \subset \Gamma_{0}(\mathfrak{n})$, whence the coverings. Furthermore, $\Gamma(\mathfrak{n})$ and $\Gamma_{2}(\mathfrak{n})$ are normal subgroups of $\mathrm{GL}_{2}(A)$, so the respective coverings are Galois with Galois groups

$$
\begin{gathered}
\operatorname{Gal}(Y(\mathfrak{n}) / \mathbb{M}) \cong \mathrm{GL}_{2}(A) / \Gamma(\mathfrak{n}) Z\left(\mathbb{F}_{q}\right) \cong \mathrm{GL}_{2}^{1}(A / \mathfrak{n}) / Z\left(\mathbb{F}_{q}\right) \quad \text { and } \\
\operatorname{Gal}\left(Y_{2}(\mathfrak{n}) / \mathbb{M}\right) \cong \mathrm{GL}_{2}(A) / \Gamma_{2}(\mathfrak{n}) \cong \operatorname{GL}_{2}^{1}(A / \mathfrak{n}) / Z^{1}(A / \mathfrak{n}) \cong \operatorname{PGL}_{2}^{1}(A / \mathfrak{n}) .
\end{gathered}
$$

Last, if every prime factor of $\mathfrak{n}$ has even degree, then every element of $\mathbb{F}_{q}$ is a square in $A / \mathfrak{n}$ and $\operatorname{PGL}_{2}^{1}(A / \mathfrak{n})=\operatorname{PSL}_{2}(A / \mathfrak{n})$.

2.4. Special subcurves of $\left(M_{A, \mathbb{C}_{\infty}}^{2}\right)^{n}$. In the Introduction we defined the notion of (pure) special subcurves of a product of $n$ Drinfeld modular curves, in particular in $\mathbb{M}^{n}$.

For a subset $I \subset\{1, \ldots, n\}$ we denote by $p_{I}: \mathbb{M}^{n} \rightarrow \mathbb{M}^{I}$ the projection onto the coordinates listed in $I$. We will also write $p_{i}:=p_{\{i\}}$ and $p_{i, j}:=p_{\{i, j\}}$.

The curve $Y_{0}(\mathfrak{n})$ parametrizes Drinfeld modules linked by a cyclic isogeny of degree $\mathfrak{n}$. If $\left(\phi, \phi^{\prime}\right)$ is such a pair, then $\phi \in \mathbb{M}\left(\mathbb{C}_{\infty}\right)$, and $\phi^{\prime} \in \mathbb{M}\left(\mathbb{C}_{\infty}\right)$ if and only if $\mathfrak{n}$ is a principal ideal. Thus, for $\mathfrak{n}=\langle N\rangle$ principal, we obtain a map $Y_{0}(\mathfrak{n}) \rightarrow \mathbb{M}^{2}$ whose image is denoted by $Y_{0}^{\prime}(N)$. Analytically, this map is given by

$$
\begin{aligned}
\Gamma_{0}(\mathfrak{n}) \backslash \Omega & \longrightarrow\left(\mathrm{GL}_{2}(A) \backslash \Omega\right) \times\left(\mathrm{GL}_{2}(A) \backslash \Omega\right), \\
{[\omega] } & \longmapsto([\omega],[N \omega]) .
\end{aligned}
$$

In particular, we see that $Y_{0}^{\prime}(N)$ is an irreducible pure special subcurve of $\mathbb{M}^{2}$. Moreover, every irreducible pure special subcurve of $\mathbb{M}^{2}$ is of the form $Y_{0}^{\prime}(N)$ for some $N \in A$. Indeed, the special curve corresponding to the map $\omega \mapsto\left(\left[g_{1}(\omega)\right],\left[g_{2}(\omega)\right]\right)$ parametrizes pairs of Drinfeld modules linked by cyclic $\mathfrak{n}$-isogenies, where

$$
\mathfrak{n}=\left\{\operatorname{det}\left(a g_{2} g_{1}^{-1}\right) \mid a \in A \text { such that all the entries of } a g_{2} g_{1}^{-1} \text { lie in } A\right\} \subset A \text {. }
$$

But since this curve lies in $\mathbb{M}^{2}$, the ideal $\mathfrak{n}$ must be principal, i.e. $\mathfrak{n}=\langle N\rangle$.

It follows that an irreducible curve $Y \subset \mathbb{M}^{n}$ is a pure special subcurve if and only if $p_{i, j}(Y)=Y_{0}^{\prime}\left(N_{i j}\right)$ for some $N_{i j} \in A$ for all $i \neq j$. 
Next, it will be convenient to study the space $\mathcal{S C}_{n}$ of all special subcurves in $\left(M_{A, \mathbb{C}_{\infty}}^{2}\right)^{n}$. Analytically, this will turn out to be the following double coset space:

$$
\mathcal{S C}_{n}\left(\mathbb{C}_{\infty}\right)^{\text {an }}:=\mathrm{GL}_{2}(k) \backslash \mathrm{GL}_{2}\left(\mathbb{A}_{f}\right)^{n} \times \Omega /\left(Z\left(\mathbb{A}_{f}\right) \mathrm{GL}_{2}(\hat{A})\right)^{n},
$$

where $\left(Z\left(\mathbb{A}_{f}\right) \mathrm{GL}_{2}(\hat{A})\right)^{n}$ acts from the right on $\mathrm{GL}_{2}\left(\mathbb{A}_{f}\right)^{n}$ in the usual way, and trivially on $\Omega$, while $\mathrm{GL}_{2}(k)$ acts from the left on $\Omega$ in the usual way, and diagonally on $\mathrm{GL}_{2}\left(\mathbb{A}_{f}\right)^{n}$.

Choose a set of representatives $T \subset \mathrm{GL}_{2}\left(\mathbb{A}_{f}\right)^{n}$ for the double quotient

$$
\mathrm{GL}_{2}(k) \backslash \mathrm{GL}_{2}\left(\mathbb{A}_{f}\right)^{n} /\left(Z\left(\mathbb{A}_{f}\right) \mathrm{GL}_{2}(\hat{A})\right)^{n} .
$$

Note that $T$ is infinite, and we again choose $1 \in T$. For each $t=\left(t_{1}, \ldots, t_{n}\right) \in T$ let $\Gamma_{t}=\bigcap_{i=1}^{n} t_{i} \mathrm{GL}_{2}(\hat{A}) t_{i}^{-1} \cap \mathrm{GL}_{2}(k)$. Then we have a canonical bijection

$$
\begin{aligned}
\mathrm{GL}_{2}(k) \backslash \mathrm{GL}_{2}\left(\mathbb{A}_{f}\right)^{n} \times \Omega /\left(Z\left(\mathbb{A}_{f}\right) \mathrm{GL}_{2}(\hat{A})\right)^{n} & \stackrel{\sim}{ } \coprod_{t \in T} \Gamma_{t} \backslash \Omega \\
{\left[\left(h_{1}, \ldots, h_{n}\right), \omega\right] } & \longmapsto\left[g^{-1}(\omega)\right]_{t},
\end{aligned}
$$

where $t \in T$ is such that $[t]=\left[\left(h_{1}, \ldots, h_{n}\right)\right]=[h]$ in (2.13), and $g \in \mathrm{GL}_{2}(k)$ is such that $h=g t a$, for some $a \in\left(Z\left(\mathbb{A}_{f}\right) \mathrm{GL}_{2}(\hat{A})\right)^{n}$. We see that $\mathcal{S C}_{n}$ is the disjoint union of an infinite family of Drinfeld modular curves, parametrized by $T$.

Next, we describe a map from $\mathcal{S C}_{n}\left(\mathbb{C}_{\infty}\right)$ into $\left(M_{A}^{2}\left(\mathbb{C}_{\infty}\right)\right)^{n}$ :

$$
\begin{aligned}
\mathcal{S C}_{n}\left(\mathbb{C}_{\infty}\right) \stackrel{\theta}{\longrightarrow} & \left(M_{A}^{2}\left(\mathbb{C}_{\infty}\right)\right)^{n} \\
\mathrm{GL}_{2}(k) \backslash \mathrm{GL}_{2}\left(\mathbb{A}_{f}\right)^{n} & \longrightarrow \mathrm{GL}_{2}(k)^{n} \backslash \mathrm{GL}_{2}\left(\mathbb{A}_{f}\right)^{n} \times \Omega^{n} /\left(Z\left(\mathbb{A}_{f}\right) \mathrm{GL}_{2}(\hat{A})\right)^{n} \\
\times \Omega /\left(Z\left(\mathbb{A}_{f}\right) \mathrm{GL}_{2}(\hat{A})\right)^{n} & =\left(\mathrm{GL}_{2}(k) \backslash \mathrm{GL}_{2}\left(\mathbb{A}_{f}\right) \times \Omega / \mathrm{GL}_{2}(\hat{A})\right)^{n} \\
{\left[\left(h_{1}, \ldots, h_{n}\right), \omega\right] } & \left(\left[h_{1}, \omega\right], \ldots,\left[h_{n}, \omega\right]\right) ; \\
\coprod_{t \in T} \Gamma_{t} \backslash \Omega & \coprod_{s \in S^{n}}\left(\Gamma_{s_{i}} \backslash \Omega\right)_{i=1}^{n} \\
{[\omega]_{t} \longmapsto } & \left(\left[g_{i}^{-1}(\omega)\right]_{s_{i}}\right)_{i=1}^{n},
\end{aligned}
$$

where $s_{i} \in S$ such that $\left[s_{i}\right]=\left[t_{i}\right]$ in $\mathrm{GL}_{2}(k) \backslash \mathrm{GL}_{2}\left(\mathbb{A}_{f}\right) / \mathrm{GL}_{2}(\hat{A})$, and $g_{i} \in \mathrm{GL}_{2}(k)$ such that $g_{i} s_{i} a_{i}=t_{i}$ for some $a_{i} \in \mathrm{GL}_{2}(\hat{A})$, for $i=1, \ldots, n$.

We note that $\mathrm{GL}_{2}(k) \backslash \mathrm{GL}_{2}\left(\mathbb{A}_{f}\right) /\left(Z\left(\mathbb{A}_{f}\right) \mathrm{GL}_{2}(\hat{A})\right)=\mathrm{GL}_{2}(k) \backslash \mathrm{GL}_{2}\left(\mathbb{A}_{f}\right) / \mathrm{GL}_{2}(\hat{A})$, as for any $x \in \mathbb{A}_{f}$ we have $\left(\begin{array}{ll}x & 0 \\ 0 & x\end{array}\right) \equiv \prod_{\mathfrak{p}}\left(\begin{array}{cc}\pi_{\mathfrak{p}}^{n_{\mathfrak{p}}} & 0 \\ 0 & \pi_{\mathfrak{p}}^{n_{\mathfrak{p}}}\end{array}\right) \bmod \operatorname{GL}_{2}(\hat{A})$, where $\pi_{\mathfrak{p}} \in k$ denotes a chosen uniformizer at $\mathfrak{p}$, and $n_{\mathfrak{p}}=\min \left(0, v_{\mathfrak{p}}(x)\right)$ is zero for almost all $\mathfrak{p}$. This latter scalar is in $\mathrm{GL}_{2}(k)$, and, as scalars commute in $\mathrm{GL}_{2}\left(\mathbb{A}_{f}\right)$, is killed by the left $\mathrm{GL}_{2}(k)$ action. The same principle does not hold when we have $n>1$ copies of $\mathrm{GL}_{2}\left(\mathbb{A}_{f}\right)$ and of $Z\left(\mathbb{A}_{f}\right) \mathrm{GL}_{2}(\hat{A})$ but only one copy of $\mathrm{GL}_{2}(k)$, which is why the $Z\left(\mathbb{A}_{f}\right)$ appears explicitly in the definition of $\mathcal{S C}_{n}\left(\mathbb{C}_{\infty}\right)^{\text {an }}$.

Let

$$
T^{0}:=\operatorname{ker}\left(T \rightarrow \mathrm{GL}_{2}(k)^{n} \backslash \mathrm{GL}_{2}\left(\mathbb{A}_{f}\right)^{n} / \mathrm{GL}_{2}(\hat{A})^{n}\right) .
$$

Then for every $t \in T^{0}$, the Drinfeld modular curve $\Gamma_{t} \backslash \Omega$ is mapped into $\mathbb{M}^{n}\left(\mathbb{C}_{\infty}\right)$ by $\theta$, where its image is a pure special curve, as defined in the Introduction. 
Conversely, all pure special curves in $\mathbb{M}^{n}$ arise in this way:

Proposition 2.15. We have bijections

$$
\begin{aligned}
& T^{0} \longleftrightarrow\left(Z(k) \mathrm{GL}_{2}(A)\right)^{n} \backslash \mathrm{GL}_{2}(k)^{n} / \mathrm{GL}_{2}(k) \longleftrightarrow\{\text { Isomorphism classes of } \\
& \text { pure special curves in } \left.\mathbb{M}^{n}\right\} \\
& t \longmapsto \quad[g]=\left[\left(g_{1}, \ldots, g_{n}\right)\right] \quad \longmapsto \quad \text { Pure special curve defined } \\
& \text { by } \\
& \omega \mapsto\left[\left(g_{1}(\omega), \ldots, g_{n}(\omega)\right)\right],
\end{aligned}
$$

with $g \in \mathrm{GL}_{2}(k)^{n}$ such that $g=a t^{-1}$ for some $a \in\left(Z\left(\mathbb{A}_{f}\right) \mathrm{GL}_{2}(\hat{A})\right)^{n}$.

Proof. In the first bijection, let $t \in T^{0}$. Then by definition there exist $g \in \mathrm{GL}_{2}(k)^{n}$ and $a^{-1} \in\left(Z\left(\mathbb{A}_{f}\right) \mathrm{GL}_{2}(\hat{A})\right)^{n}$ such that $g t a^{-1}=1$. Then $[g]=\left[a t^{-1}\right]$ is welldefined, since if also $g^{\prime}=a^{\prime} t^{-1}$, then $g^{\prime}=a^{\prime} a^{-1} g$ with $a^{\prime} a^{-1} \in\left(Z\left(\mathbb{A}_{f}\right) \mathrm{GL}_{2}(\hat{A})\right)^{n} \cap$ $\mathrm{GL}_{2}(k)^{n}=\left(Z(k) \mathrm{GL}_{2}(A)\right)^{n}$. Moreover, given $g \in \mathrm{GL}_{2}(k)^{n}$, there exist $h \in \mathrm{GL}_{2}(k)$, $t \in T^{0}$ and $a \in\left(Z\left(\mathbb{A}_{f}\right) \mathrm{GL}_{2}(\hat{A})\right)^{n}$ such that $g^{-1}=h t a$, hence the map is surjective. If also $g^{-1}=h^{\prime} t^{\prime} a^{\prime}$, then it is clear that $t=t^{\prime}$, by definition of $T$.

The second bijection follows as $\left(g_{1}, \ldots, g_{n}\right)$ and $\left(g_{1}^{\prime}, \ldots, g_{n}^{\prime}\right)$ in $\mathrm{GL}_{2}(k)^{n}$ define the same pure special curve in $\mathbb{M}^{n}$ if and only if $g_{i}^{\prime}=\gamma_{i} g_{i} \sigma$ for all $i=1, \ldots, n$, for some $\left(\gamma_{1}, \ldots, \gamma_{n}\right) \in\left(Z(k) \mathrm{GL}_{2}(A)\right)^{n}$ and $\sigma \in \mathrm{GL}_{2}(k)$.

This proposition justifies our definition of $\mathcal{S C}_{n}$ as the space of all pure special subcurves of $\left(M_{A, \mathbb{C}_{\infty}}^{2}\right)^{n}$. We also denote by $\mathcal{S C}_{n}^{0} \subset \mathcal{S C}_{n}$ the subfamily of those Drinfeld modular curves corresponding to $T^{0}$, i.e. $\mathcal{S C}_{n}^{0}\left(\mathbb{C}_{\infty}\right)^{\text {an }} \cong \coprod_{t \in T^{0}} \Gamma_{t} \backslash \Omega$, which is the space of all pure special subcurves of $\mathbb{M}^{n}$.

It remains to define special subvarieties of $\left(M_{A, \mathbb{C}_{\infty}}^{2}\right)^{n}$ as products of pure special subcurves and CM points.

2.5. Pure special curves and trees. Let $\mathfrak{p} \subset A$ be a prime. Recall (e.g. [16]) that the Brûhat-Tits tree of $\mathrm{GL}_{2}\left(k_{\mathfrak{p}}\right)$ is the $(|\mathfrak{p}|+1)$-regular tree whose vertices represent homothety classes of $A_{\mathfrak{p}}$-lattices in $k_{\mathfrak{p}}^{2}$, and two vertices representing classes $\Lambda$ and $\Lambda^{\prime}$ are joined by an (unoriented) edge if there exist representative lattices $L \in \Lambda$ and $L^{\prime} \in \Lambda^{\prime}$ such that $L^{\prime} \subset L$ and $L / L^{\prime} \cong A / \mathfrak{p}$. We denote by $\mathcal{T}_{\mathfrak{p}}$ the set of vertices of this tree. The group $\mathrm{GL}_{2}\left(k_{\mathfrak{p}}\right)$ acts transitively on $\mathcal{T}_{\mathfrak{p}}$, and the stabilizer of the "origin" $v_{o, \mathfrak{p}}$, which is the vertex corresponding to the lattice $A_{\mathfrak{p}}^{2}$, is $Z\left(k_{\mathfrak{p}}\right) \mathrm{GL}_{2}\left(A_{\mathfrak{p}}\right)$. Thus we get a bijection $\mathcal{T}_{\mathfrak{p}} \leftrightarrow \mathrm{GL}_{2}\left(k_{\mathfrak{p}}\right) / Z\left(k_{\mathfrak{p}}\right) \mathrm{GL}_{2}\left(A_{\mathfrak{p}}\right)$.

Hence we have bijections

$$
T \longleftrightarrow \mathrm{GL}_{2}(k) \backslash \mathrm{GL}_{2}\left(\mathbb{A}_{f}\right)^{n} /\left(Z\left(\mathbb{A}_{f}\right) \mathrm{GL}_{2}(\hat{A})\right)^{n} \longleftrightarrow \mathrm{GL}_{2}(k) \backslash \prod_{\mathfrak{p}}^{\prime} \mathcal{T}_{\mathfrak{p}}^{n} .
$$

Here the restricted product $\prod_{\mathfrak{p}}^{\prime}$ denotes the subset of those families $\left(v_{1, \mathfrak{p}}, \ldots, v_{n, \mathfrak{p}}\right)_{\mathfrak{p}}$ of $n$-tuples of vertices $v_{i, \mathfrak{p}} \in \mathcal{T}_{\mathfrak{p}}$ such that $v_{1, \mathfrak{p}}=\cdots=v_{n, \mathfrak{p}}=v_{o, \mathfrak{p}}$ for almost all primes $\mathfrak{p}$.

We also have the bijection

$$
\mathrm{GL}_{2}(k) \backslash \prod_{\mathfrak{p}}^{\prime} \mathcal{T}_{\mathfrak{p}}^{n} \longleftrightarrow S \times \prod_{\mathfrak{p}}^{\prime} \mathrm{GL}_{2}\left(k_{\mathfrak{p}}\right) \backslash \mathcal{T}_{\mathfrak{p}}^{n} \quad\left(\leftrightarrow \operatorname{Pic}(A) \times \prod_{\mathfrak{p}}^{\prime} \mathrm{GL}_{2}\left(k_{\mathfrak{p}}\right) \backslash \mathcal{T}_{\mathfrak{p}}^{n}\right)
$$


which arises from

$$
\begin{gathered}
\mathrm{GL}_{2}(k) \backslash \mathrm{GL}_{2}\left(\mathbb{A}_{f}\right)^{n} /\left(Z\left(\mathbb{A}_{f}\right) \mathrm{GL}_{2}(\hat{A})\right)^{n} \\
\stackrel{\sim}{\longrightarrow} \mathrm{GL}_{2}(k) \backslash \mathrm{GL}_{2}\left(\mathbb{A}_{f}\right) / Z\left(\mathbb{A}_{f}\right) \mathrm{GL}_{2}(\hat{A}) \times \mathrm{GL}_{2}\left(\mathbb{A}_{f}\right) \backslash \mathrm{GL}_{2}\left(\mathbb{A}_{f}\right)^{n} /\left(Z\left(\mathbb{A}_{f}\right) \mathrm{GL}_{2}(\hat{A})\right)^{n}, \\
{\left[g_{1}, \ldots, g_{n}\right] \longmapsto\left(\left[g_{1}\right],\left[1, g_{1}^{-1} g_{2}, \ldots, g_{1}^{-1} g_{n}\right]\right),}
\end{gathered}
$$

which is readily verified. This bijection may also be of help to the reader puzzling over [3, §1.3].

The moduli interpretation of (2.16) and (2.17) is the following.

We may view $\mathcal{T}_{\mathfrak{p}}$ as the tree of $\mathfrak{p}$-isogenies of Drinfeld modules as in the elliptic curve case, but with the added subtlety that different vertices will map to different irreducible components of $\left(M_{A, \mathbb{C}_{\infty}}^{2}\right)^{n}$ when $\mathfrak{p} \subset A$ is not principal. Now let $t \in$ $T$ correspond to the pure special subcurve $C_{t} \stackrel{\theta}{\hookrightarrow}\left(M_{A, \mathbb{C}_{\infty}}^{2}\right)^{n}$. Let $(s, \underline{v}) \in S \times$ $\prod_{\mathfrak{p}}{ }^{\prime} \mathrm{GL}_{2}(k) \backslash \mathcal{T}_{\mathfrak{p}}{ }^{n}$ correspond to $t$ via (2.17). We may choose a representative family $\left(v_{1, \mathfrak{p}}, \ldots, v_{n, \mathfrak{p}}\right)_{\mathfrak{p}}$ of the class $\underline{v}$ such that $v_{1, \mathfrak{p}}=v_{o, \mathfrak{p}}$ for all $\mathfrak{p}$. Then a typical point $x=\left(x_{1}, \ldots, x_{n}\right) \in C_{t}\left(\mathbb{C}_{\infty}\right)$ satisfies: $x_{1}$ lies on the $s$-component of $M_{A, \mathbb{C}_{\infty}}^{2}$, and the isogenies $x_{1} \rightarrow x_{2}, x_{1} \rightarrow x_{3}, \ldots, x_{1} \rightarrow x_{n}$ correspond to $\left(v_{2, \mathfrak{p}}, \ldots, v_{n, \mathfrak{p}}\right)_{\mathfrak{p}}$, respectively.

In the case $n=3$ we have a particularly pleasing combinatorial description of $\mathrm{GL}_{2}(k) \backslash \mathcal{T}_{\mathfrak{p}}^{3}$. Any triple of vertices $\left(v_{1}, v_{2}, v_{3}\right) \in \mathcal{T}_{\mathfrak{p}}^{3}$ has a well-defined center $v_{c}$, defined by the property that the three paths (possibly of length zero) from $v_{c}$ to the $v_{i}$ are pairwise edge-disjoint. Denote by $\left(n_{1}, n_{2}, n_{3}\right) \in \mathbb{N}_{0}^{3}$ the lengths of these paths. Furthermore, $\mathrm{GL}_{2}\left(k_{\mathfrak{p}}\right)$ acts 3 -transitively on the set $\mathbb{P}^{1}\left(k_{\mathfrak{p}}\right)$ of ends of $\mathcal{T}_{\mathfrak{p}}$, and it follows that a triple of vertices $\left(v_{1}, v_{2}, v_{3}\right)$ with corresponding center $v_{c}$ and triple of path-lengths $\left(n_{1}, n_{2}, n_{3}\right)$ is mapped by $\mathrm{GL}_{2}(k)$ to another triple $\left(v_{1}^{\prime}, v_{2}^{\prime}, v_{3}^{\prime}\right)$ with center $v_{c}^{\prime}$ and path-lengths $\left(n_{1}^{\prime}, n_{2}^{\prime}, n_{3}^{\prime}\right)$ if and only if $\left(n_{1}, n_{2}, n_{3}\right)=\left(n_{1}^{\prime}, n_{2}^{\prime}, n_{3}^{\prime}\right)$. See also [3, §1.3]. Thus we have bijections

$$
\mathrm{GL}_{2}(k) \backslash \mathcal{T}_{\mathfrak{p}}^{3} \longleftrightarrow \mathbb{N}_{0}^{3}, \quad \prod_{\mathfrak{p}}^{\prime} \mathrm{GL}_{2}(k) \backslash \mathcal{T}_{\mathfrak{p}}^{3} \longleftrightarrow \mathcal{I}_{A}^{3}, \quad T \longleftrightarrow \operatorname{Pic}(A) \times \mathcal{I}_{A}^{3},
$$

where $\mathcal{I}_{A}$ denotes the semigroup of non-zero $A$-ideals. The second bijection is given by

$$
\left(v_{1, \mathfrak{p}}, v_{2, \mathfrak{p}}, v_{3, \mathfrak{p}}\right)_{\mathfrak{p}} \mapsto\left(n_{1, \mathfrak{p}}, n_{2, \mathfrak{p}}, n_{3, \mathfrak{p}}\right)_{\mathfrak{p}} \mapsto\left(\mathfrak{n}_{1}, \mathfrak{n}_{2}, \mathfrak{n}_{3}\right)=\prod_{\mathfrak{p}}\left(\mathfrak{p}^{n_{1, \mathfrak{p}}}, \mathfrak{p}^{n_{2, \mathfrak{p}}}, \mathfrak{p}^{n_{3, \mathfrak{p}}}\right) .
$$

Let $Y$ be the special curve corresponding to the data $\left([\mathfrak{a}],\left(\mathfrak{n}_{1}, \mathfrak{n}_{2}, \mathfrak{n}_{3}\right)\right) \in \operatorname{Pic}(A) \times$ $\mathcal{I}_{A}^{3}$. Then $Y$ maps into $\mathbb{M}^{3}$ if and only if $[\mathfrak{a}]=1$ and $\mathfrak{n}_{i}=\left\langle N_{i}\right\rangle$ is principal for $i=1,2,3$. In this case we have $p_{i, j}(Y)=Y_{0}^{\prime}\left(N_{i} N_{j}\right) \subset \mathbb{M}^{2}$. In particular, we have shown

Lemma 2.19. The set of pure special subcurves of $\mathbb{M}^{3}$ is in bijection with $\left(A / \mathbb{F}_{q}^{\times}\right)^{3}$.

In general, if a pure modular curve $Y \subset\left(M_{A, \mathbb{C}_{\infty}}^{2}\right)^{3}$ corresponds to a triple $\left(\mathfrak{n}_{1}, \mathfrak{n}_{2}, \mathfrak{n}_{3}\right) \in \mathcal{I}_{A}^{3}$, then $p_{i, j}(Y)$ is isomorphic to a suitable irreducible component of $M_{A}^{2}\left(\mathcal{K}_{0}\left(\mathfrak{n}_{i} \mathfrak{n}_{j}\right)\right)_{\mathbb{C}_{\infty}}$.

The following result will be important later on. 
Proposition 2.20. Let $Y \subset \mathbb{M}^{3}$ be a pure special curve corresponding to a triple $\left(\mathfrak{n}_{1}, \mathfrak{n}_{2}, \mathfrak{n}_{3}\right)$, and fix $\left(x_{1}, x_{2}\right) \in p_{1,2}\left(Y\left(\mathbb{C}_{\infty}\right)\right)$. Then

$$
\left|p_{1,2}^{-1}\left(x_{1}, x_{2}\right) \cap Y\left(\mathbb{C}_{\infty}\right)\right| \geq \prod_{\mathfrak{p} \mid \mathfrak{n}_{3}} \frac{(|\mathfrak{p}|-1)(|\mathfrak{p}|+1)^{n_{3, \mathfrak{p}}-1}}{2 n_{3, \mathfrak{p}}+1},
$$

where $\mathfrak{n}_{i}=\prod_{\mathfrak{p}} \mathfrak{p}^{n_{i, \mathfrak{p}}}$.

Proof. Let $\left(v_{1, \mathfrak{p}}, v_{2, \mathfrak{p}}, v_{3, \mathfrak{p}}\right)_{\mathfrak{p}} \in \prod_{\mathfrak{p}}^{\prime} \mathcal{T}_{\mathfrak{p}}^{3}$ be a representative family of tuples corresponding to the special curve $Y$, and denote by $\left(v_{c, \mathfrak{p}}\right)_{\mathfrak{p}}$ its family of centers. Fixing $x_{1}$ and $x_{2}$ amounts to fixing $v_{1, \mathfrak{p}}, v_{2, \mathfrak{p}}$ for all $\mathfrak{p}$. We now count the possible vertex families $\left(v_{3, \mathfrak{p}}\right)_{\mathfrak{p}}$ for which $\left(v_{1, \mathfrak{p}}, v_{2, \mathfrak{p}}, v_{3, \mathfrak{p}}\right)_{\mathfrak{p}}$ represents $Y$. As $\left(\mathfrak{n}_{1}, \mathfrak{n}_{2}, \mathfrak{n}_{3}\right)$ is prescribed, so is $v_{c, \mathfrak{p}}$ for every $\mathfrak{p}$, and from (2.18) it follows that we must count the paths of length $n_{3, \mathfrak{p}}$ from $v_{c, \mathfrak{p}}$ which are edge-disjoint from the two paths leading to $v_{1, \mathfrak{p}}$ and $v_{2, \mathfrak{p}}$, which gives at least $(|\mathfrak{p}|-1)(|\mathfrak{p}|+1)^{n_{3, \mathfrak{p}}-1}$ for each $\mathfrak{p}$. Thus the number of valid cyclic $\mathfrak{n}_{3}$-isogenies from $x_{c}$ (corresponding to $\left.\left(v_{c, \mathfrak{p}}\right)_{\mathfrak{p}}\right)$ to $x_{3}$ is given by $\prod_{\mathfrak{p} \mid \mathfrak{n}_{3}}(|\mathfrak{p}|-1)(|\mathfrak{p}|+1)^{n_{3, \mathfrak{p}}-1}$.

But if $x_{c}$ has complex multiplication by an order in $K$, then distinct isogenies may well lead to the same $x_{3}$; they correspond to non-trivial endomorphisms $f \in \operatorname{End}\left(x_{c}\right)$ of norm $N_{K / k}(f)=\mathfrak{n}_{3}^{2}$. There are at most $\prod_{\mathfrak{p}}\left(2 n_{3, \mathfrak{p}}+1\right)$ such endomorphisms, which completes the proof.

2.6. Main results and reduction to $\mathbb{M}^{n}$. Let $\mathcal{K}_{i} \subset \mathrm{GL}_{2}(\hat{A})$ be subgroups of finite index, for $i=1, \ldots, n$. With a bit more effort, we could give a general treatment of pure special subcurves of $\prod_{i=1}^{n} M_{A}^{2}\left(\mathcal{K}_{i}\right)_{\mathbb{C}_{\infty}}$ as in the previous two sections. But the following definition is much easier:

Consider the morphism

$$
\pi: \prod_{i=1}^{n} M_{A}^{2}\left(\mathcal{K}_{i}\right)_{\mathbb{C}_{\infty}} \longrightarrow\left(M_{A, \mathbb{C}_{\infty}}^{2}\right)^{n}
$$

induced by the inclusions $\mathcal{K}_{i} \hookrightarrow \mathrm{GL}_{2}(\hat{A})$. Then an irreducible subvariety $Y \subset$ $\prod_{i=1}^{n} M_{A}^{2}\left(\mathcal{K}_{i}\right)_{\mathbb{C}_{\infty}}$ is a special subvariety if and only if the image $\pi(Y)$ is a special subvariety of $\left(M_{A, \mathbb{C}_{\infty}}^{2}\right)^{n}$. Similarly for (pure) special subcurves.

We point out that this definition is equivalent to the one given in 1.2 . Intuitively, a special subvariety is one defined by imposing isogeny relations between coordinates, and setting other coordinates equal to $\mathrm{CM}$ values.

We restate our main result as follows.

Theorem 1.2. Let $\mathcal{K}_{i} \subset \mathrm{GL}_{2}(\hat{A})$ be subgroups of finite index for $i=1, \ldots, n$. Let $Y \subset \prod_{i=1}^{n} M_{A}^{2}\left(\mathcal{K}_{i}\right)_{\mathbb{C}_{\infty}}$ be an irreducible subvariety. Then $Y\left(\mathbb{C}_{\infty}\right)$ contains a Zariski-dense subset of CM points if and only if $Y$ is a special subvariety.

As every irreducible component of $M_{A}^{2}\left(\mathcal{K}_{i}\right)_{\mathbb{C}_{\infty}}$ is a Drinfeld modular curve corresponding to $\Gamma_{s} \backslash \Omega$, for the arithmetic subgroup $\Gamma_{s}=s \mathcal{K}_{i} s^{-1} \cap \mathrm{GL}_{2}(k)$ with some $s \in \mathrm{GL}_{2}\left(\mathbb{A}_{f}\right)$, we see that the above theorem is equivalent to Theorem 1.2

Next we want to show that it suffices to prove Theorem 1.2 for $X=\mathbb{M}^{n}$.

Proposition 2.21. Let $\Gamma_{i}^{\prime} \subset \Gamma_{i} \subset \mathrm{GL}_{2}(k)$ be arithmetic subgroups corresponding to Drinfeld modular curves $X_{i}^{\prime}$ and $X_{i}$, respectively, for $i=1, \ldots, n$. Let $X=$ $X_{1} \times \cdots \times X_{n}$ and $X^{\prime}=X_{1}^{\prime} \times \cdots \times X_{n}^{\prime}$. Then the canonical map $f: X^{\prime} \rightarrow X$ preserves special subvarieties. In other words, a subvariety $Y \subset X^{\prime}$ is special if and only if $f(Y)$ is special. 
Proof. This is immediate, as special subvarieties are defined purely in terms of isogeny relations between coordinates, and the property that any constant projections $Y \rightarrow X_{i}^{\prime}$ have CM points as images. These properties are independent of any level structures.

Now let $\Gamma_{i} \subset \mathrm{GL}_{2}(k)$ be an arithmetic subgroup such that $X_{i}\left(\mathbb{C}_{\infty}\right)^{\text {an }} \cong \Gamma_{i} \backslash \Omega$, for each $i=1, \ldots, n$. Then each $\Gamma_{i}^{\prime}=\mathrm{GL}_{2}(A) \cap \Gamma_{i}$ is again an arithmetic subgroup, and we have maps

$$
\Gamma_{i}^{\prime} \backslash \Omega \longrightarrow \Gamma_{i} \backslash \Omega, \quad \Gamma_{i}^{\prime} \backslash \Omega \longrightarrow \mathrm{GL}_{2}(A) \backslash \Omega
$$

corresponding to morphisms of Drinfeld modular curves

$$
f_{i}: X_{i}^{\prime} \longrightarrow X_{i}, \quad g_{i}: X_{i}^{\prime} \longrightarrow \mathbb{M},
$$

for each $i=1, \ldots, n$.

Applying Proposition 2.21 to the maps $\prod_{i} f_{i}$ and $\prod_{i} g_{i}$ shows that Theorem 1.2 holds for $X_{1} \times \cdots \times X_{n}$ if and only if it holds for $\mathbb{M}^{n}$.

With this reduction step out of the way, the rest of our proof of Theorem 1.2 will follow [3] very closely, with $\mathbb{M}^{n}$ playing the role of $\mathbb{A}^{n}$. The main geometric aspect of $\mathbb{A}^{n}$ used in $[3$ is its structure as the product of affine lines, and it turns out that the product structure of $\mathbb{M}^{n}$ will suffice, with some caveats. First, $\mathbb{M}$ is not defined over $k$ but over $H$, the Hilbert class field of $k$. (Classically, the elliptic modular curve $Y(1)$ is defined over the Hilbert class field of $\mathbb{Q}$, which is just $\mathbb{Q}$ itself. In our case, $k \neq H$ in general, which illustrates the "metamathematical" phenomenon of splitting of the two different roles of $\mathbb{Q}$ into two different fields ( $k$ and $H$ ) in characteristic $p$. See [9, Preface] for a discussion.)

Secondly, in general the isomorphism

$$
\mathrm{GL}_{2}(A) \backslash \Omega \stackrel{\sim}{\longrightarrow} \mathbb{M}\left(\mathbb{C}_{\infty}\right)^{\text {an }}
$$

is no longer induced by a well-behaved " $j$-invariant" $j: \Omega \rightarrow \mathbb{C}_{\infty}$, as is the case for $A=\mathbb{F}_{q}[T]$. Consequently, we cannot take advantage of the analytic properties of the $j$-function, and will say nothing about the Weil heights of CM points.

\section{HECKE CORRESPONDENCES AND CM POINTS}

3.1. General formalism. We briefly describe Hecke correspondences on $M_{A}^{2}(\mathcal{K})$ in general. Let $g \in \mathrm{GL}_{2}\left(\mathbb{A}_{f}\right)$, and set $\mathcal{K}_{g}=\mathcal{K} \cap g^{-1} \mathcal{K} g$. Then $g$ acts from the left on $M_{A}^{2}\left(\mathcal{K}_{g}\right)$ (by letting $g^{-1}$ act from the right on Drinfeld modules with full level structures; see [7, II.3]). Combined with the standard projection $\pi: M_{A}^{2}\left(\mathcal{K}_{g}\right) \rightarrow$ $M_{A}^{2}(\mathcal{K})$, this gives rise to the Hecke correspondence on $M_{A}^{2}(\mathcal{K})$ :

$$
T_{g}: M_{A}^{2}(\mathcal{K}) \stackrel{\pi}{\longleftarrow} M_{A}^{2}\left(\mathcal{K}_{g}\right) \stackrel{\pi \circ g}{\longrightarrow} M_{A}^{2}(\mathcal{K}) .
$$

This is an algebraic correspondence of finite degree $\left[\mathcal{K}: \mathcal{K}_{g}\right]$.

Analytically, this correspondence is described as follows. The group $\mathrm{GL}_{2}\left(\mathbb{A}_{f}\right)$ acts from the left on $\mathrm{GL}_{2}\left(\mathbb{A}_{f}\right) \times \Omega$ by $g \cdot(h, \omega)=\left(h g^{-1}, \omega\right)$. This gives rise to the correspondence

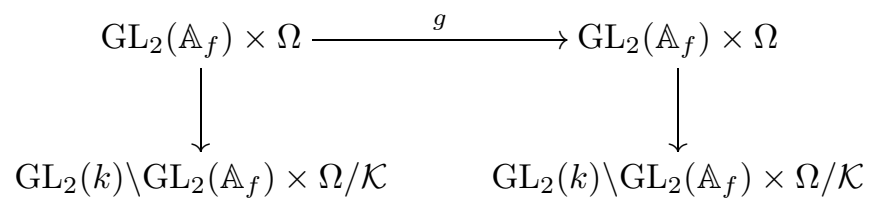


which is easily seen to factor through $\mathrm{GL}_{2}(k) \times \mathcal{K}_{g}$, thus describing the finite correspondence

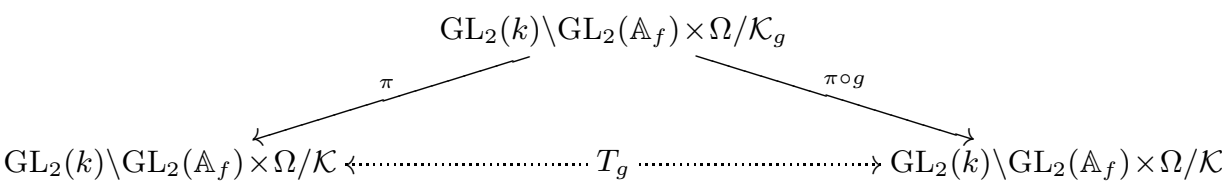

Note that if $\mathcal{K}_{g}$ is a congruence subgroup, then $T_{g}$ is defined over the class field corresponding to the class group $K^{\times} \backslash \mathbb{A}_{f}^{\times} / \operatorname{det}\left(\mathcal{K}_{g}\right)$.

If $\operatorname{det}\left(\mathcal{K}_{g}\right)=\operatorname{det}(\mathcal{K})$, then the canonical projection $M_{A}^{2}\left(\mathcal{K}_{g}\right) \rightarrow M_{A}^{2}(\mathcal{K})$ induces a bijection between the sets of irreducible components of $M_{A}^{2}\left(\mathcal{K}_{g}\right)_{\mathbb{C}_{\infty}}$ and $M_{A}^{2}(\mathcal{K})_{\mathbb{C}_{\infty}}$, respectively, and in this case we say that $T_{g}$ is irreducible on $M_{A}^{2}(\mathcal{K})$.

Suppose that $T_{g}$ is irreducible on $M_{A}^{2}(\mathcal{K})$. We will describe the action of $T_{g}$ on $\coprod_{s \in S} \Gamma_{s} \backslash \Omega$ explicitly.

Let $[\omega] \in \Gamma_{s} \backslash \Omega$; this lifts to $(s, \omega) \in \mathrm{GL}_{2}\left(\mathbb{A}_{f}\right) \times \Omega$, and is sent to $\left(s g^{-1}, \omega\right)$ by $T_{g}$. Now let $s_{g, s} \in S, f_{g, s} \in \mathrm{GL}_{2}(k)$ and $k_{g, s} \in \mathcal{K}$ such that

$$
f_{g, s} \cdot s g^{-1} \cdot k_{g, s}=s_{g, s} \in S
$$

It follows that the element $\left[s g^{-1}, \omega\right] \in \mathrm{GL}_{2}(k) \backslash \mathrm{GL}_{2}\left(\mathbb{A}_{f}\right) \times \Omega / \mathcal{K}$ equals $\left[s_{g, s}, f_{g, s}(\omega)\right]$, and thus maps to $\left[f_{g, s}(\omega)\right] \in \Gamma_{s_{g, s}} \backslash \Omega$ via (2.6). Thus we see that $T_{g}$ maps the $s$-component to the $s_{g, s}$-component, and the restriction of $T_{g}$ to $\Gamma_{s} \backslash \Omega$ is the correspondence induced by $f_{g, s}$ :

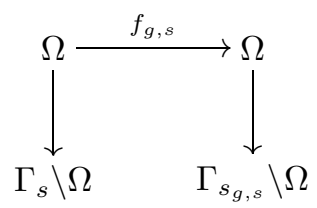

Choose a set $\left\{t_{i} \in \mathrm{GL}_{2}(k) \mid i \in I_{g, s}\right\}$ of representatives for the left quotient space $\Gamma_{s_{g, s}} \backslash \Gamma_{s_{g, s}} f_{g, s} \Gamma_{s}$. Then it follows that the action of $T_{g}$ on $\Gamma_{s} \backslash \Omega$ is given explicitly by

$$
T_{g}:[\omega] \longmapsto \bigcup_{i \in I_{g, s}}\left[t_{i}(\omega)\right] \subset \Gamma_{s_{g, s}} \backslash \Omega .
$$

From the bijection (2.7) we see that $\operatorname{det}\left(s_{g, s}\right)=\operatorname{det}(s) \operatorname{det}(g)^{-1} \bmod k^{\times} \operatorname{det}(\mathcal{K})$, so if $\operatorname{det}(g) \in k^{\times} \operatorname{det}(\mathcal{K})$, then $T_{g}$ preserves the irreducible components of $M_{A}^{2}(\mathcal{K})_{\mathbb{C}_{\infty}}$.

3.2. Hecke correspondences on $\mathbb{M}^{n}$. From now on, we will assume that $\mathcal{K}=$ $\mathrm{GL}_{2}(\hat{A})$ and $g=\left(\begin{array}{cc}N & 0 \\ 0 & 1\end{array}\right)$ for some $N \in A$ with $|N|>1$. In this case, we see that $\mathcal{K}_{g}=\mathcal{K}_{0}(\langle N\rangle)=: \mathcal{K}_{0}(N)$. The Hecke correspondence $T_{g}$ is irreducible on $M_{A}^{2}$ and preserves its irreducible components. $T_{g}$ restricted to $\mathbb{M}$ factors through the main component of $M_{A}^{2}\left(\mathcal{K}_{0}(N)\right)_{\mathbb{C}_{\infty}}$, which is $\Gamma_{0}(N) \backslash \Omega$. Moreover, setting $s=1$ in (3.3) 
we get $f_{g, 1}=g$, and so from (3.4) it follows that $T_{N}:=\left.T_{g}\right|_{\mathbb{M}}$ is given by

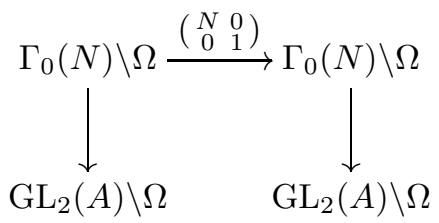

The moduli interpretation of $T_{N}$ is that it maps a Drinfeld module $\phi$ to the set of all Drinfeld modules linked to $\phi$ via cyclic $N$-isogenies. It follows that $T_{N}$ is also described by the inclusion $Y_{0}^{\prime}(N) \subset \mathbb{M}^{2}$ from (2.11). The correspondence $T_{N}$ therefore coincides with what we called $T_{\mathfrak{m}}$ in [3]. In the remainder of this section, we closely follow [3, §2].

We define $T_{\mathbb{M}^{n}, N}$ to be the correspondence on $\mathbb{M}^{n}$ which is the product of the correspondences $T_{N}$ on each factor $\mathbb{M}$. When there is no risk of confusion, we also denote it by $T_{N}$. We say $T_{\mathbb{M}^{n}, N}$ stabilizes an algebraic subvariety $Y \subset \mathbb{M}^{n}$ if $Y \subset T_{\mathbb{M}^{n}, N}(Y)$. In this case, we define the restriction of $T_{N}$ to $Y$, denoted $T_{Y, N}$, to be the union of the irreducible components of $T_{\mathbb{M}^{n}, N} \cap(Y \times Y)$ of maximal dimension. It is a correspondence on $Y$ which is still surjective, in the sense that the two projections $T_{Y, N} \rightarrow Y$ are surjective. Whenever we mention $T_{Y, N}$, it is implicit that $T_{N}$ stabilizes $Y$.

Let $x \in \mathbb{M}\left(\mathbb{C}_{\infty}\right)$, and suppose that $x \in T_{N}(x)$. Then $x$ admits a cyclic endomorphism, hence is a CM point. For a given $N \in A,|N|>1$, there are only finitely many points stabilized by $T_{N}$, which correspond to the points of the diagonal in $\mathbb{M}^{2}$ which intersect $Y_{0}^{\prime}(N)$. On the other hand, given a $\mathrm{CM}$ point $x \in \mathbb{M}\left(\mathbb{C}_{\infty}\right)$ with $R=\operatorname{End}(x)$, there are infinitely many $N \in A$ such that $x \in T_{N}(x)$, namely all those $N$ composed of primes $\mathfrak{p} \subset A$ for which $\mathfrak{p} R$ is a product of two distinct principal prime ideals of $R$. Equivalently, these primes split completely in the class field corresponding to $\operatorname{Pic}(R)$, hence the set of such primes has density $1 / 2|\operatorname{Pic}(R)|$, by Cebotarev. Note also that such primes $\mathfrak{p}$ are necessarily principal in $A$.

3.3. Some intersection theory. $\mathbb{M}$ is affine, so we may fix an embedding $\mathbb{M} \subset$ $\mathbb{A}_{\mathbb{C}_{\infty}}^{m}$. Then we obtain embeddings $\mathbb{M}^{n} \subset \mathbb{A}_{\mathbb{C}_{\infty} n}^{m n}$ and $\overline{\mathbb{M}^{n}} \subset \mathbb{P}_{\mathbb{C}_{\infty}}^{m n}$. (Here ${ }^{-}$denotes the Zariski-closure.)

For any irreducible $Y \subset \mathbb{M}^{n}$, we denote by $\operatorname{deg}(Y)$ the degree of $\bar{Y} \subset \mathbb{P}^{m n}$ in the usual sense. If $Y=\bigcup_{i} Y_{i}$ is a union of irreducible components, then $\operatorname{deg}(Y):=$ $\sum_{i} \operatorname{deg}\left(Y_{i}\right)$ (regardless of their dimensions).

We denote by $\psi(N)=\left[\mathrm{GL}_{2}(A): \Gamma_{0}(N)\right]=|N| \prod_{\mathfrak{p} \mid N}\left(1+|\mathfrak{p}|^{-1}\right)$ the degree of the correspondence $T_{N}$ on $\mathbb{M}$. In particular, $\psi(N) \leq \operatorname{deg}\left(Y_{0}^{\prime}(N)\right) \leq 2 \psi(N)$.

We collect the following facts.

Proposition 3.7. Let $Y \subset \mathbb{M}^{n}$ be an algebraic subvariety.

(1) $Y$ has at most $\operatorname{deg}(Y)$ irreducible components.

(2) If $Y^{\prime} \subset \mathbb{M}^{n}$ is another subvariety, then $\operatorname{deg}\left(Y \cap Y^{\prime}\right) \leq \operatorname{deg}(Y) \operatorname{deg}\left(Y^{\prime}\right)$.

(3) $\operatorname{deg}\left(T_{\mathbb{M}^{n}, N}(Y)\right) \leq 2^{n} \psi(N)^{n} \operatorname{deg}(Y)$.

(4) There are only finitely many pure special subcurves of $\mathbb{M}^{n}$ of degree less than a given bound.

Proof. (1) is trivial, (2) and (3) follow from [6, 8.4.6], and (4) follows from the fact that a curve $Y \subset \mathbb{M}^{n}$ is pure special if and only if $p_{i, j}(Y)=Y_{0}^{\prime}\left(N_{i j}\right)$ for some $N_{i j} \in A$ for all $i \neq j$, and $\operatorname{deg}\left(Y_{0}^{\prime}\left(N_{i j}\right)\right) \geq \psi\left(N_{i j}\right)$. 
3.4. Preimages in $\Omega^{n}$. The group $\mathrm{GL}_{2}\left(k_{\infty}\right)^{n}$ acts on $\Omega^{n}$. Denote by $\pi$ the rigid analytic map $\pi: \Omega^{n} \rightarrow \mathbb{M}^{n}\left(\mathbb{C}_{\infty}\right)^{\text {an }}$, which is the quotient for the $\mathrm{GL}_{2}(A)^{n}$-action.

Let $Y_{i}$ be an irreducible component of $Y$. Then by [14, Kor 3.5] $Y_{i}\left(\mathbb{C}_{\infty}\right)^{\text {an }}$ is irreducible as a rigid analytic variety. Choose an irreducible rigid analytic subvariety $Z_{i} \subset \pi^{-1}\left(Y_{i}\right)$ such that $\pi\left(Z_{i}\right)=Y_{i}$. Now since $\mathrm{GL}_{2}(A)^{n}$ acts transitively on the fibres of $\pi$, it follows that the $\mathrm{GL}_{2}(A)^{n}$-orbit of $Z_{i}$ is all of $\pi^{-1}\left(Y_{i}\right)$. We next describe the action of $T_{N}$ in $\Omega^{n}$.

Set $\mathcal{I}=\{1, \ldots, \psi(N)\}$, and let $\left\{t_{j} \in \mathrm{GL}_{2}(k)^{n} \mid j \in \mathcal{I}^{n}\right\}$ be a set of representatives for $\left(\mathrm{GL}_{2}(A) \backslash \mathrm{GL}_{2}(A)\left(\begin{array}{cc}N & 0 \\ 0 & 1\end{array}\right) \mathrm{GL}_{2}(A)\right)^{n}$.

For each $Z_{i}$ we define $\mathcal{J}_{Z_{i}} \subset \mathcal{I}^{n}$ as the set of those indices $j$ for which $t_{j}\left(Z_{i}\right) \subset$ $\pi^{-1}\left(Y_{i}\right)$. Now let $y \in Y_{i}\left(\mathbb{C}_{\infty}\right)$ and choose some $z \in Z_{i}$ with $\pi(z)=y$. Then by (3.5) we get

$$
\begin{aligned}
T_{\mathbb{M}^{n}, N}(y) & =\left\{\pi\left(t_{j}(z)\right) \mid j \in \mathcal{I}^{n}\right\}, \\
T_{Y, N}(y) & =\left\{\pi\left(t_{j}(z)\right) \mid j \in \mathcal{J}_{Z_{i}}\right\} .
\end{aligned}
$$

In particular, we see that each $\mathcal{J}_{Z_{i}}$ is non-empty, as $T_{Y, N}$ is a surjective correspondence. In fact, under suitable conditions, the sets $\mathcal{J}_{Z_{i}}$ are fairly large.

Proposition 3.10. Let $Y \subset \mathbb{M}^{n}$ be a subvariety all of whose irreducible components have the same dimension, and suppose that $Y \subset T_{\mathbb{M}^{n}, N}(Y)$ for some $N \in A$ such that $\langle N\rangle$ is a product of distinct primes $\mathfrak{p} \subset A$ of even degree satisfying $|\mathfrak{p}| \geq$ $\max (13, \operatorname{deg}(Y))$. Let $I \subset\{1, \ldots, n\}$ and let $Y_{i}$ be an irreducible component of $Y$ for which the projection $p_{I}: Y_{i} \rightarrow \mathbb{M}^{I}$ is dominant, and choose a preimage $Z_{i} \subset \Omega^{n}$ of $Y_{i}\left(\mathbb{C}_{\infty}\right)^{\text {an }}$. Then the projection

$$
p_{I}: \mathcal{J}_{Z_{i}} \longrightarrow \mathcal{I}^{I}
$$

is surjective.

Proof. This is [3, Thm. 4]; the proof is exactly the same. We briefly explain the hypotheses on $N$ : we use the fact that $\mathbb{M}$ has a $\operatorname{PSL}_{2}(A / N A) \cong \prod_{\mathfrak{p}} \operatorname{PSL}_{2}(A / \mathfrak{p})$ covering $Y_{2}(N)$, of which $Y_{0}(N)$ is a subcover (Proposition 2.10), and that this group has no proper subgroups of index $\operatorname{deg}(Y)$ or less when $|\mathfrak{p}| \geq \max (13, \operatorname{deg}(Y))$.

3.5. An interlude in group theory. We remark that the $\mathrm{GL}_{2}\left(k_{\infty}\right)$ action on $\Omega$ induces a $\mathrm{PGL}_{2}\left(k_{\infty}\right)$ action, as the center acts trivially. Until now we have found it more convenient to work with $\mathrm{GL}_{2}$, but in order to continue we will need a number of group-theoretic results, and here it will be simpler to work with $\mathrm{PGL}_{2}$, as had been done throughout 3 .

For the convenience of the reader, we recall here some basic properties of the groups $\mathrm{PGL}_{2}\left(k_{\infty}\right)$, which we will need. These results were used implicitly in [3, and thus may also help the reader with that paper.

Lemma 3.11. $k_{\infty}^{\times} / k_{\infty}^{\times 2} \cong(\mathbb{Z} / 2 \mathbb{Z})^{2}$.

Proof. This is trivial, as $k_{\infty} \cong \mathbb{F}_{q}((\varpi))$ for a uniformizer $\varpi$, and $q$ is odd.

Proposition 3.12. Every non-trivial normal subgroup of $\mathrm{PGL}_{2}\left(k_{\infty}\right)$ contains $\mathrm{PSL}_{2}\left(k_{\infty}\right)$. In particular, $\mathrm{PGL}_{2}\left(k_{\infty}\right)$ has no non-trivial discrete normal subgroups.

Proof. Let $H \triangleleft \mathrm{PGL}_{2}\left(k_{\infty}\right)$ be a normal subgroup. Then, as $\mathrm{PSL}_{2}\left(k_{\infty}\right)$ is simple, either $\mathrm{PSL}_{2}\left(k_{\infty}\right) \subset H$, or $H \cap \mathrm{PSL}_{2}\left(k_{\infty}\right)=\{1\}$. In the latter case we get an embedding $H \hookrightarrow \mathrm{PGL}_{2}\left(k_{\infty}\right) / \mathrm{PSL}_{2}\left(k_{\infty}\right) \cong k_{\infty}^{\times} / k_{\infty}^{\times 2} \cong(\mathbb{Z} / 2 \mathbb{Z})^{2}$. It remains to show 
that $\mathrm{PGL}_{2}\left(k_{\infty}\right)$ has no normal subgroups isomorphic to $\mathbb{Z} / 2 \mathbb{Z}$ or $(\mathbb{Z} / 2 \mathbb{Z})^{2}$. This may be verified with explicit calculations, by conjugating elements of order 2 with $\left(\begin{array}{ll}1 & 1 \\ 0 & 1\end{array}\right)$.

Proposition 3.13. Let $H$ be a subgroup of finite index in $G=\mathrm{PGL}_{2}\left(k_{\infty}\right)$. Then $H$ is normal and contains $\mathrm{PSL}_{2}\left(k_{\infty}\right)$. In particular, if $H$ is simple, then $H=$ $\operatorname{PSL}_{2}\left(k_{\infty}\right)$.

Proof. $G$ acts on the cosets $G / H$, giving a representation $\rho: G \rightarrow \operatorname{Aut}(G / H) \cong S_{n}$ where $n=[G: H]$. Then $K=\operatorname{ker}(\rho)$ is a normal subgroup of $G$ contained in $H$. As $K$ is infinite, $K$ must intersect non-trivially with $\mathrm{PSL}_{2}\left(k_{\infty}\right)$, hence contains $\mathrm{PSL}_{2}\left(k_{\infty}\right)$, as the latter is simple. We will not need the fact that $H$ is normal, but it is known that all the subgroups between $\operatorname{PSL}_{2}\left(k_{\infty}\right)$ and $\mathrm{PGL}_{2}\left(k_{\infty}\right)$ are normal in $G$.

Corollary 3.14. Let $\mathrm{PSL}_{2}\left(k_{\infty}\right) \subset H \subset \mathrm{PGL}_{2}\left(k_{\infty}\right)$ and suppose that $f: H \hookrightarrow$ $\mathrm{PGL}_{2}\left(k_{\infty}\right)$ is a monomorphism with image of finite index. Then $\left.f\right|_{\mathrm{PSL}_{2}\left(k_{\infty}\right)}$ in an automorphism of $\mathrm{PSL}_{2}\left(k_{\infty}\right)$.

Proof. The image $f\left(\mathrm{PSL}_{2}\left(k_{\infty}\right)\right)$ has finite index in $\mathrm{PGL}_{2}\left(k_{\infty}\right)$ and is simple, hence $f\left(\mathrm{PSL}_{2}\left(k_{\infty}\right)\right)=\mathrm{PSL}_{2}\left(k_{\infty}\right)$.

The above results may easily be generalized to $\mathrm{PGL}_{2}$ over other (infinite) fields. Mostly we have just used the fact that $k_{\infty}^{\times} / k_{\infty}^{\times 2}$ is finite.

Proposition 3.15 (Goursat's Lemma). Let $G_{1}$ and $G_{2}$ be groups, and $H \subset G_{1} \times G_{2}$ a subgroup such that the two projections $\mathrm{pr}_{i}: H \rightarrow G_{i}$ are surjective. Then $K_{i}=$ $\operatorname{ker}\left(p_{i}\right)$ can be considered a normal subgroup of $G_{j}$, for $i \neq j$, and $H$ is the inverse image of the graph of an isomorphism $\rho: G_{1} / K_{2} \stackrel{\sim}{\rightarrow} G_{2} / K_{1}$.

Proof. This is straightforward. The map

$$
\begin{aligned}
\rho: G_{1} / K_{2} & \longrightarrow G_{2} / K_{1}, \\
g_{1} & \longmapsto g_{2} \text { with }\left(g_{1}, g_{2}\right) \in H
\end{aligned}
$$

is easily checked to be a well-defined isomorphism. Now $H$ is the inverse image of the graph of $\rho$.

Remark 3.16. In the situation of Proposition 3.15, if $H^{\prime} \subset H$, then $\rho$ induces an isomorphism $H^{\prime} K_{2} / K_{2} \stackrel{\sim}{\longrightarrow} H^{\prime} K_{1} / K_{1}$.

3.6. Curves stabilized by Hecke correspondences. Our next goal is to characterize special subvarieties of $\mathbb{M}^{n}$ by their property of being stabilized by suitable Hecke correspondences. The hard part is to prove this for curves.

Theorem 3.17. Let $Y \subset \mathbb{M}^{2}$ be an irreducible algebraic curve such that both projections $Y \rightarrow \mathbb{M}$ are dominant, and suppose $Y \subset T_{\mathbb{M}^{2}, P}(Y)$ for a principal prime $\mathfrak{p}=\langle P\rangle \subset A$ of even degree satisfying $|\mathfrak{p}| \geq \max (13, \operatorname{deg}(Y))$. Then $Y=Y_{0}^{\prime}\left(N^{\prime}\right)$ for some $N^{\prime} \in A$.

Note that we cannot deduce $N^{\prime}$ from $P$. Indeed $Y_{0}^{\prime}\left(N^{\prime}\right)$ is stabilized by $T_{\mathbb{M}^{2}, N}$ for all $N \in A$ coprime to $N^{\prime}$. 
Proof. Suppose that the hypotheses of Theorem 3.17 are satisfied. The group $G:=$ $\mathrm{PGL}_{2}\left(k_{\infty}\right)^{2}$ acts on $\Omega^{2}$, and we define the following subgroups: $S:=\mathrm{PSL}_{2}\left(k_{\infty}\right)^{2}$, $\Gamma:=\mathrm{PGL}_{2}(A)^{2}$ and $\Sigma:=\mathrm{PSL}_{2}(A)^{2}$. We fix an irreducible rigid analytic curve $Z \subset \Omega^{2}$ with $\pi(Z)=Y\left(\mathbb{C}_{\infty}\right)^{\text {an }}$. Let $G_{Z} \subset G$ be the stabilizer of $Z$, which is a closed analytic subgroup of $G$. We also define the subgroups $S_{Z}=G_{Z} \cap S$, $\Gamma_{Z}=G_{Z} \cap \Gamma$ and $\Sigma_{Z}=G_{Z} \cap \Sigma$. We intend to prove Theorem 3.17 by investigating the structure of $G_{Z}$ and $S_{Z}$.

Denote by $p r_{i}: G_{Z} \rightarrow \mathrm{PGL}_{2}\left(k_{\infty}\right)$ the two projections for $i=1,2$.

Lemma 3.18. The projection $\mathrm{pr}_{i}: G_{Z} \rightarrow \mathrm{PGL}_{2}\left(k_{\infty}\right)$ is injective, and $p r_{i}\left(\Gamma_{Z}\right)$ has finite index in $\mathrm{PGL}_{2}(A)$, for $i=1,2$.

Proof. Exactly the same as [3, Lemma 2.11]. Here one uses Proposition 3.12

We choose representatives $\left\{t_{i} \in \mathrm{GL}_{2}(k)^{2} \mid i \in \mathcal{I}^{2}\right\}$ for

$$
\left(\mathrm{GL}_{2}(A) \backslash \mathrm{GL}_{2}(A)\left(\begin{array}{ll}
P & 0 \\
0 & 1
\end{array}\right) \mathrm{GL}_{2}(A)\right)^{2}
$$

such that both components of the $t_{i}$ are of the form

$$
\left(\begin{array}{ll}
P & 0 \\
0 & 1
\end{array}\right) \quad \text { or } \quad\left(\begin{array}{ll}
1 & b \\
0 & P
\end{array}\right) \text { for representatives } b \text { for } A / \mathfrak{p} .
$$

Let $j \in \mathcal{J}_{Z}$; then $t_{j}(Z) \subset \pi^{-1}(Y)$, so there is some $\gamma_{j} \in \Gamma$ such that $\gamma_{j} t_{j} \in G_{Z}$. As the projections $p r_{1}, p r_{2}: \mathcal{J}_{Z} \rightarrow \mathcal{I}$ are surjective (Proposition 3.10), we get many non-trivial elements of $G_{Z}$ this way.

Let $H_{1}=p r_{1}\left(G_{Z}\right)$. Our first goal is to show that $H_{1}$ contains $\mathrm{PSL}_{2}\left(k_{\infty}\right)$. By a slight abuse of notation, we also denote the elements $p r_{1}\left(t_{i}\right) \in \mathrm{GL}_{2}(k)$ by $t_{i}$, with $i \in \mathcal{I}$. We have seen that, for every $i \in p r_{1}\left(\mathcal{J}_{Z}\right)=\mathcal{I}$, there exists some $\gamma_{i} \in \mathrm{PGL}_{2}(A)$ such that $g_{i}:=\gamma_{i} t_{i} \in H_{1}$. Lemma 3.18 says that $H_{1} \cap \mathrm{PGL}_{2}(A)$ has finite index in $\mathrm{PGL}_{2}(A)$, and we choose a finite set $R \subset \mathrm{PGL}_{2}(A)$ of representatives for $\mathrm{PGL}_{2}(A) /\left(\mathrm{PGL}_{2}(A) \cap H_{1}\right)$.

We claim that given any string $i_{1}, \ldots, i_{n}$ of elements of $\mathcal{I}$, and any $a \in \mathrm{GL}_{2}(A)$, there exists $\gamma \in R$ such that

$$
\gamma t_{i_{n}} t_{i_{n-1}} \cdots t_{i_{1}} a \in H_{1} .
$$

Indeed, by induction it suffices to prove the claim for $n=1$. Let $i_{1} \in \mathcal{I}$ and $a \in \mathrm{GL}_{2}(A)$ be given. $\mathrm{GL}_{2}(A)$ acts from the right on the set of left cosets $\mathrm{GL}_{2}(A) \backslash \mathrm{GL}_{2}(A)\left(\begin{array}{ll}P & 0 \\ 0 & 1\end{array}\right) \mathrm{GL}_{2}(A)$, so we let $a$ act from the right on $\mathrm{GL}_{2}(A) \cdot t_{i_{1}}$, obtaining $\mathrm{GL}_{2}(A) \cdot t_{i_{1}} a=\mathrm{GL}_{2}(A) \cdot t_{j}$ for some $j \in \mathcal{I}$. Thus $t_{i_{1}} a=\gamma_{j}^{\prime} t_{j}$, and for suitable $\gamma \in R$ and $\gamma^{\prime} \in H_{1} \cap \operatorname{PGL}_{2}(A)$ we have

$$
\gamma t_{i_{1}} a=\gamma \gamma_{j}^{\prime} t_{j}=\gamma\left(\gamma_{j}^{\prime} \gamma_{j}^{-1}\right) \gamma_{j} t_{j}=\gamma^{\prime} \gamma_{j} t_{j}=\gamma^{\prime} g_{j} \in H_{1} .
$$

This proves the claim.

Now, multiplying by a suitable power of $\left(\begin{array}{ll}P & 0 \\ 0 & P\end{array}\right)$, we see from (3.19) that for any $x \in A[1 / P]$ and $a \in \mathrm{GL}_{2}(A)$, there exists some $\gamma_{x, a} \in R$ such that $\gamma_{x, a}\left(\begin{array}{ll}1 & x \\ 0 & 1\end{array}\right) a \in H_{1}$. Denote by $E \subset \operatorname{PSL}_{2}(A[1 / P])$ the subgroup generated by elementary matrices. We have shown that $H_{1} \cap E$ has finite index in $E$. As $A[1 / P]$ is dense in $k_{\infty}$, and $\mathrm{PSL}_{2}\left(k_{\infty}\right)$ is generated by elementary matrices, it follows that $E$ is dense in $\mathrm{PSL}_{2}\left(k_{\infty}\right)$.

Next, we see that $H_{1}$ is a closed subgroup of $\mathrm{PGL}_{2}\left(k_{\infty}\right)$, exactly the same way as [3, Lemma 2.12 and the following paragraph], where we need only replace 
$\operatorname{PSL}_{2}(A[1 / \mathfrak{m}])$ by $E$. It follows that $H_{1} \cap \mathrm{PSL}_{2}\left(k_{\infty}\right)$ has finite index in $\operatorname{PSL}_{2}\left(k_{\infty}\right)$, so by Proposition 3.13

$$
\mathrm{PSL}_{2}\left(k_{\infty}\right) \subset H_{1}, \quad \text { and similarly, } \mathrm{PSL}_{2}\left(k_{\infty}\right) \subset H_{2}=p r_{2}\left(G_{Z}\right) .
$$

Now, since the projections $p r_{1}, p r_{2}: G_{Z} \rightarrow \mathrm{PGL}_{2}\left(k_{\infty}\right)$ are injective, Proposition 3.15 implies that

$$
G_{Z}=\left\{(g, \rho(g)) \mid g \in H_{1}\right\},
$$

from some isomorphism $\rho: H_{1} \stackrel{\sim}{\rightarrow} H_{2}$. From $(3.20$ ) and Corollary 3.14 it follows that

$$
S_{Z}=G_{Z} \cap \mathrm{PSL}_{2}\left(k_{\infty}\right)^{2}=\left\{(g, \rho(g)) \mid g \in \mathrm{PSL}_{2}\left(k_{\infty}\right)\right\},
$$

where $\left.\rho\right|_{\mathrm{PSL}_{2}\left(k_{\infty}\right)}$ is an automorphism of $\mathrm{PSL}_{2}\left(k_{\infty}\right)$ (see also Remark 3.16).

It is known that the automorphisms of $\mathrm{PSL}_{2}\left(k_{\infty}\right)$ are all of the form $g \mapsto h g^{\sigma} h^{-1}$ for some $h \in \mathrm{PGL}_{2}\left(k_{\infty}\right)$ and $\sigma \in \operatorname{Aut}\left(k_{\infty}\right)$; see [12. By the definition of $\Sigma_{Z}$ and (3.21), we see that $h \cdot p r_{1}\left(\Sigma_{Z}\right)^{\sigma} \cdot h^{-1} \subset \mathrm{PSL}_{2}(A)$. On the other hand, Lemma 3.18 tells us that $\operatorname{pr}_{1}\left(\Sigma_{Z}\right)$ and $h \cdot \operatorname{pr}_{1}\left(\Sigma_{Z}\right)^{\sigma} \cdot h^{-1}$ have finite index in $\operatorname{PSL}_{2}(A)$. This in turn tells us a lot about $h$ and $\sigma$.

Following the proof of [3, Prop. 2.13], we get

Proposition 3.22. Let $G$ be a subgroup of finite index in $\mathrm{PGL}_{2}(A)$, and suppose that $h G^{\sigma} h^{-1}$ is also a subgroup of finite index in $\mathrm{PGL}_{2}(A)$, for some $h \in \mathrm{PGL}_{2}\left(k_{\infty}\right)$ and $\sigma \in \operatorname{Aut}\left(k_{\infty}\right)$. Then $\sigma$ induces an automorphism of $k$ which fixes $\infty$, and $h \in \mathrm{PGL}_{2}(k)$.

Remark 3.23. The statement of [3, Prop. 2.13] is false as it stands; one needs to add the hypothesis that $h G^{\sigma} h^{-1}$ is a subgroup of finite index in $\mathrm{PGL}_{2}(A)$. This hypothesis is satisfied in the situation where the proposition is applied. The author would like to thank the anonymous referee for pointing out this omission.

Now that we have assembled enough ingredients, the proof of Theorem 3.17 follows just as in [3, §2.7]. We provide a sketch. We have seen that

$$
S_{Z}=\left\{\left(g, h g^{\sigma} h^{-1}\right) \mid g \in \mathrm{PSL}_{2}\left(k_{\infty}\right)\right\},
$$

where $h \in \mathrm{PGL}_{2}(k)$ and $\sigma \in \operatorname{Aut}\left(k_{\infty}\right)$ is as in Proposition 3.22 .

Denote by $C / \mathbb{F}_{q}$ a smooth irreducible projective curve such that $k=\mathbb{F}_{q}(C)$ and $A=\Gamma\left(C-\{\infty\}, \mathcal{O}_{C}\right)$, and write $q=p^{n}$. Then $\sigma^{n}$ fixes $C$ and the point $\infty \in C$, and it follows that $\sigma$ has finite order. Thus $\sigma$ fixes $\mathbb{F}_{q}$, and there exists an integer $t \geq 0$ such that $\sigma(\alpha)=\alpha^{p^{t}}$, for all $\alpha \in \mathbb{F}_{q}$.

Let $F \subset k_{\infty}$ be the fixed field of $\sigma$, so $\left[k_{\infty}: F\right]$ is finite. Fix some non-square $\alpha \in \mathbb{F}_{q}$, and set

$$
\mathcal{P}=\left\{z \in \Omega \mid z^{2}=\alpha e, e \in F\right\} \subset \Omega .
$$

Pick any $z_{1} \in \mathcal{P}$. Then $S_{1}=\operatorname{Stab}_{\mathrm{PSL}_{2}(F)}\left(z_{1}\right)$ is a one-dimensional Lie group.

Now let $z_{2} \in \Omega$ such that $\left(z_{1}, z_{2}\right) \in Z$. Then the " $S_{1}$-orbit"

$$
\left\{\left(g\left(z_{1}\right), h g^{\sigma} h^{-1}\left(z_{2}\right)\right) \mid g \in S_{1}\right\} \subset Z \cap\left(\left\{z_{1}\right\} \times \Omega\right)
$$

is discrete, but $S_{1}$ is not, so there exists $1 \neq g \in S_{1}$ such that $h g^{\sigma} h^{-1}$ fixes both $z_{2}$ and $h^{\prime}\left(z_{1}\right)$, where $h^{\prime}=h \circ\left(\begin{array}{cc}\alpha^{\left(p^{t}-1\right) / 2} & 0 \\ 0 & 1\end{array}\right) \in \mathrm{PGL}_{2}(k)$. This means that $z_{2}$ and $h^{\prime}\left(z_{1}\right)$ are conjugate over $k_{\infty}$, i.e. $z_{2}= \pm h^{\prime}\left(z_{1}\right)$. It follows that either $\pi\left(z_{1}, h^{\prime}\left(z_{1}\right)\right) \in Y\left(\mathbb{C}_{\infty}\right)$ or $\pi\left(-z_{1}, h^{\prime}\left(-z_{1}\right)\right) \in Y\left(\mathbb{C}_{\infty}\right)$. Both of these points also lie on $Y_{0}^{\prime}\left(N^{\prime}\right)$, where $N^{\prime}$ is the degree of the cyclic isogeny represented by $h^{\prime}$, and is 
independent of $\left(z_{1}, z_{2}\right)$. As $\mathcal{P}$ is uncountable, whereas the fibers of $\pi$ are countable, it follows that $Y=Y_{0}^{\prime}\left(N^{\prime}\right)$.

To extend Theorem 3.17 to subvarieties $Y \subset \mathbb{M}^{n}$ of higher dimension we may follow [3, §2.8 and Corollaries 2.8 and 2.9] almost verbatim; no new ingredients are required.

Theorem 3.24. Denote by $H$ the Hilbert class field of $k$, and let $F$ be a field lying between $H$ and $\mathbb{C}_{\infty}$. Let $Y \subset \mathbb{M}^{n}$ be an $F$-irreducible algebraic subvariety, containing a $C M$ point $x \in Y\left(\mathbb{C}_{\infty}\right)$. Suppose that $Y \subset T_{\mathbb{M}^{n}, N}(Y)$ for some $N \in A$ such that $\langle N\rangle$ is a product of distinct primes $\mathfrak{p} \subset A$ of even degree satisfying $|\mathfrak{p}| \geq$ $\max (13, \operatorname{deg}(Y))$. Then $Y \subset \mathbb{M}^{n}$ is a special subvariety.

3.7. CM points on curves. We may now start proving our main results by exploiting the behavior of CM points under Galois action (Proposition 2.1) in conjunction with Theorems 3.17 and 3.24. We first treat the case of curves, where our results are effective.

Theorem 3.25. Let $X=X_{1} \times \cdots \times X_{n}$ be a product of Drinfeld modular curves. Let $F / H$ be a finite extension, and $d \in \mathbb{N}$. Then there exists an absolutely computable constant $B=B(X, F, d)>0$ such that the following holds. Let $Y \subset X$ be an irreducible algebraic subcurve of degree $d$ and defined over $F$. Then $Y$ is a special subcurve if and only if $Y\left(\mathbb{C}_{\infty}\right)$ contains a $C M$ point $x$ satisfying $H_{\mathrm{CM}}(x)>B$.

This indeed implies Theorem 1.2 for curves, since if $Y \subset X$ contains a Zariskidense set of CM points, then by Proposition 2.3 it contains CM points of arbitrary $\mathrm{CM}$ height and $Y$ is defined over some finite extension $F / k$, as the CM points are all defined over $k^{\text {sep }}$.

Proof. We again follow [3, §3.4] very closely, but will provide full details here for the benefit of the reader.

It follows from Proposition 2.21 that we may assume that $X=\mathbb{M}^{n}$. Furthermore, we may assume that none of the projections $p_{i}: Y \rightarrow \mathbb{M}$ are constant. Using the fact that $Y \subset \mathbb{M}^{n}$ is a pure special subcurve if and only if $p_{i, j}(Y) \subset \mathbb{M}^{2}$ is special for all $i<j$, we have reduced the problem to the case $n=2$.

Let $x=\left(x_{1}, x_{2}\right) \in Y\left(\mathbb{C}_{\infty}\right)$ be a CM point. For $i=1,2$ we write $R_{i}=\operatorname{End}\left(x_{i}\right)=$ $A+\mathfrak{f}_{i} \mathcal{O}_{K_{i}}$, an order of conductor $\mathfrak{f}_{i} \subset A$ in the CM field $K_{i}$, which has genus $g_{i}$. Furthermore, we denote by $K_{i}\left(x_{i}\right):=H_{R_{i}}$ the ring class field of $R_{i}$, which is a field of definition for $x_{i}$, and we have $\operatorname{Gal}\left(K_{i}\left(x_{i}\right) / K_{i}\right) \cong \operatorname{Pic}\left(R_{i}\right)$, and $K_{i}\left(x_{i}\right) / K_{i}$ is unramified outside $\mathfrak{f}_{i}$. We denote by $K=K_{1} K_{2}$ and $K\left(x_{1}, x_{2}\right)=K_{1}\left(x_{1}\right) K_{2}\left(x_{2}\right)$ the composite fields. Denote by $F_{s}$ the separable closure of $k$ in $F$ (which contains $H)$, and by $L$ the Galois closure of $F_{s} K\left(x_{1}, x_{2}\right)$ over $k$.

Let $\mathfrak{p}$ be a prime of $k$ of even degree which splits completely in $F_{s} K$ (in particular, $\mathfrak{p}$ is principal, as it splits in $H$ ), and suppose $\mathfrak{p} \nmid \mathfrak{f}_{1} \mathfrak{f}_{2}$. Let $\mathfrak{P}$ be a prime of $L$ lying above $\mathfrak{p}$, and denote by $\mathfrak{P}_{i}$ its restriction to $K_{i}\left(x_{i}\right)$. Denote by $\sigma \in \operatorname{Aut}(F L / F K)$ an extension of the Frobenius element $(\mathfrak{P}, L / k)$, and let $\sigma_{i}=\left.\sigma\right|_{K_{i}\left(x_{i}\right)}=\left(\mathfrak{P}_{i}, K_{i}\left(x_{i}\right) / K_{i}\right)$ (remember that $\mathfrak{p}$ splits in $K$ and is unramified in $L)$.

It follows from Proposition 2.1 that $x_{i}$ and $x_{i}^{\sigma}$ correspond to Drinfeld modules linked by cyclic $\mathfrak{p}$-isogenies, and hence

$$
\left(x_{1}, x_{2}\right) \in Y \cap T_{\mathbb{M}^{2}, \mathfrak{p}}\left(Y^{\sigma}\right)=Y \cap T_{\mathbb{M}^{2}, \mathfrak{p}}(Y) .
$$


Moreover, the whole $\operatorname{Gal}\left(F K\left(x_{1}, x_{2}\right) / F\right)$-orbit of $\left(x_{1}, x_{2}\right)$ lies in this intersection, which thus contains at least $\max \left(\left|\operatorname{Pic}\left(R_{1}\right)\right|,\left|\operatorname{Pic}\left(R_{2}\right)\right|\right) /[F: k]$ points. On the other hand, from Proposition 3.7] $\operatorname{deg}\left(Y \cap T_{\mathbb{M}^{2}, \mathfrak{p}}(Y)\right) \leq 4(|\mathfrak{p}|+1)^{2} \operatorname{deg}(Y)^{2}$. Therefore, if

$$
\left|\operatorname{Pic}\left(R_{i}\right)\right| /[F: k]>4(|\mathfrak{p}|+1)^{2} \operatorname{deg}(Y)^{2}, \quad \text { for } i=1 \text { or } 2,
$$

then the intersection is improper, $Y \subset T_{\mathbb{M}^{2}, \mathfrak{p}}(Y)$ and hence $Y$ is special (Theorem 3.17), provided also $|\mathfrak{p}| \geq \max (13, \operatorname{deg}(Y))$.

It remains to show that such a suitable prime $\mathfrak{p}$ indeed exists if $H_{\mathrm{CM}}(x)$ is sufficiently large. Let $M$ be the Galois closure of $F_{s} K$ over $k$, and set

$$
\pi_{M}(t)=\#\left\{\mathfrak{p} \subset A \mid \text { prime, split in } M \text { and }|\mathfrak{p}|=q^{t}\right\} .
$$

Let $T \in k$ be a transcendental element such that $k$ is a finite separable geometric extension of $\mathbb{F}_{q}(T)$, and let $e=\left[k: \mathbb{F}_{q}(T)\right]$. Let $\mathbb{F}$ be the algebraic closure of $\mathbb{F}_{q}$ in $M$, let $n_{c}=\left[\mathbb{F}: \mathbb{F}_{q}\right]$ be the constant extension degree and let $n_{g}=[M: \mathbb{F} k]$ be the geometric extension degree of $M / k$. The Čebotarev Theorem for function fields $[5$, Prop. 5.16] says

$$
\text { if } n_{c} \mid t \text {, then }\left|\pi_{M}(t)-\frac{1}{n_{g}} q^{t} / t\right|<4\left(e^{2}+g_{M}(e+1) / 2+g_{k}+1\right) q^{t / 2},
$$

where $g_{M}$ and $g_{k}$ are the genera of $M$ and $k$, respectively. We may bound $g_{M}$ in terms of $g_{1}, g_{2}$ and $g_{k}$ using the Castelnuovo inequality [17, III.10.3], and eventually obtain

$$
\pi_{M}(t)>C_{1} q^{t} / t-\left(C_{2}\left(g_{1}+g_{2}\right)+C_{3}\right) q^{t / 2},
$$

where $C_{1}, C_{2}$ and $C_{3}$ are absolutely computable positive constants, depending on $k$ and $F$.

We want $\pi_{M}(t)>\log _{q}\left|\mathfrak{f}_{1} \mathfrak{f}_{2}\right|, q^{t} \geq \max (13, \operatorname{deg}(Y))$ and $2 n_{c} \mid t$, so that there exists a prime $\mathfrak{p}$ which splits in $M$ (and thus in $F_{s} K$ ), does not divide $\mathfrak{f}_{1} \mathfrak{f}_{2}$, and satisfies the hypotheses of Theorem 3.17. We also want (3.27) to hold, for which we employ Proposition 2.4.

$$
\left|\operatorname{Pic}\left(R_{i}\right)\right|>C_{\varepsilon} H_{\mathrm{CM}}\left(x_{i}\right)^{1-\varepsilon}=C_{\varepsilon}\left(q^{g_{i}}\left|\mathfrak{f}_{i}\right|\right)^{1-\varepsilon}, \quad \text { for any } \varepsilon>0 .
$$

In summary, we need a simultaneous solution $t \in 2 n_{c} \mathbb{N}$ to the three inequalities

$$
\begin{gathered}
q^{t} \geq \max (13, \operatorname{deg}(Y)), \\
C_{1} q^{t} / t-\left(C_{2}\left(g_{1}+g_{2}\right)+C_{3}\right) q^{t / 2}>\log _{q}\left|\mathfrak{f}_{1} \mathfrak{f}_{2}\right|,
\end{gathered}
$$

and

$$
C_{\varepsilon}\left(q^{g_{i}}\left|\mathfrak{f}_{i}\right|\right)^{1-\varepsilon}>4[F: k]\left(q^{t}+1\right)^{2} \operatorname{deg}(Y)^{2} \quad \text { for some } \varepsilon>0 \text {, and } i=1 \text { or } 2 .
$$

Such a solution will always exist if $H_{\mathrm{CM}}(x)=\max \left(H_{\mathrm{CM}}\left(x_{1}\right), H_{\mathrm{CM}}\left(x_{2}\right)\right)$ is sufficiently large. (Intuitively, $q^{t}$ must be large compared to $\log _{q}\left|\mathfrak{f}_{i}\right|$ and $g_{i}$, and small compared to $\left|\mathfrak{f}_{i}\right|$ and $q^{g_{i}}$.)

3.8. Completing the proof of Theorem 1.2. The proof of Theorem 1.2 now follows exactly as in [3, §3.5]. We sketch the proof here for the sake of completeness.

First, as $Y\left(\mathbb{C}_{\infty}\right)$ contains a Zariski-dense subset $\mathcal{S}$ of $\mathrm{CM}$ points, which are defined over $k^{\text {sep }}$, there exists a finite Galois extension $F / k$ over which $Y$ is defined. We may assume that $F$ contains the Hilbert class field $H$ of $k$. We will use induction on $d=\operatorname{dim}(Y)$, the case $d=1$ following from Theorem 3.25 so we assume that 
$d \geq 2$ and $n \geq 3$. We may furthermore assume that $Y \subset \mathbb{M}^{n}$ is a hypersurface, as it is an irreducible component of

$$
\bigcap_{\substack{I \subset\{1, \ldots, n\} \\|I|=d+1}} p_{I}^{-1} p_{I}(Y) .
$$

Last, we may assume that all the projections $p_{i}: Y \rightarrow \mathbb{M}$ are dominant.

Step 1. For a given constant $B>0$, we may assume that every $x=\left(x_{1}, \ldots, x_{n}\right)$ $\in \mathcal{S}$ satisfies $H_{\mathrm{CM}}\left(x_{i}\right)>B, \forall i=1, \ldots, n$ (otherwise we replace $\mathcal{S}$ by a Zariski-dense subset).

Pick one such $x \in \mathcal{S}$. Suppose that there exist primes $\mathfrak{p}_{1}, \ldots, \mathfrak{p}_{d-1} \subset A$ of even degree satisfying the following conditions:

(i) Each $\mathfrak{p}_{j}$ splits in $F$ and in $\operatorname{End}\left(x_{i}\right)$, for all $i=1, \ldots, n$.

(ii) $\left|\mathfrak{p}_{1}\right| \geq \max (13, \operatorname{deg}(Y))$.

(iii) $\left|\mathfrak{p}_{j+1}\right| \geq(\operatorname{deg}(Y))^{2^{j}} \prod_{m=1}^{j}\left(2\left|\mathfrak{p}_{m}\right|+2\right)^{n 2^{j-m}}$, for $j=1, \ldots, d-2$.

(iv) $\left|\operatorname{Pic}\left(\operatorname{End}\left(x_{i}\right)\right)\right|>[F: k]\left|\mathfrak{p}_{d-1}\right|^{2}\left(2\left|\mathfrak{p}_{d-1}\right|+2\right)^{n}$, for all $i=1, \ldots, n$.

Note that the $\mathfrak{p}_{i}$ are principal, as they split in $H$. Then one shows, again using Galois action on $x$ together with Theorem 3.24 that there exists a pure special subvariety $Y_{x} \subset Y$ containing $x$.

As the points in $\mathcal{S}$ are Zariski-dense, it follows that there exists a Zariski-dense family $\mathcal{C}$ of pure special subcurves $C \subset Y, C \in \mathcal{C}$. (Clearly any pure special subvariety contains pure special subcurves.)

Step 2. We now show that $Y$ is special. Choose a CM point $x_{1} \in \mathbb{M}\left(\mathbb{C}_{\infty}\right)$, and consider the slice

$$
Y_{1}=Y \cap\left(\left\{x_{1}\right\} \times \mathbb{M}^{n-1}\right) .
$$

Each pure special curve $C \in \mathcal{C}$ intersects $Y_{1}$ in at least one CM point, and we denote by $Y^{\prime}$ the Zariski-closure of these intersection points:

$$
Y^{\prime}=\overline{\bigcup_{C \in \mathcal{C}}\left(C \cap Y_{1}\right)} \subset Y_{1} .
$$

We must have $\operatorname{dim}\left(Y^{\prime}\right)<\operatorname{dim}(Y)$, so by the induction hypothesis, $Y^{\prime}$ is special. We write $Y^{\prime}=Y_{1}^{\prime} \cup \cdots \cup Y_{r}^{\prime}$ as a union of irreducible components. Replacing $\mathcal{C}$ by a Zariski-dense subfamily and renumbering if necessary, we may assume that $Y_{1}^{\prime}$ contains at least $1 / r$ of the points of $C \cap Y_{1}$ for all $C \in \mathcal{C}$.

Now, either $Y_{1}^{\prime} \cong\{y\} \times \mathbb{M}^{m}$ for some CM point $y \in \mathbb{M}^{n-m}\left(\mathbb{C}_{\infty}\right)$, in which case $Y=Y_{0}^{\prime}\left(N^{\prime}\right) \times \mathbb{M}^{n-2}$ is special (here we use the fact that Theorem 1.2 has already been proved for curves), or else at least one pure special curve appears as a factor of $Y_{1}^{\prime}$.

It follows that there exist indices $1<i<j$ such that $p_{i, j}\left(Y_{1}^{\prime}\right)=Y_{0}^{\prime}(M)$ for some fixed $M \in A$. Fix $C \in \mathcal{C}$ and let the pure special curve $p_{\{1, i, j\}}(C) \subset \mathbb{M}^{3}$ correspond to the triple $\left(N_{C, 1}, N_{C, i}, N_{C, j}\right) \in A^{3}$ via Lemma 2.19 Again restricting $\mathcal{C}$ and switching $i$ and $j$ if necessary, we may assume that $\left|N_{C, i}\right| \leq\left|N_{C, j}\right|$ for all $C \in \mathcal{C}$.

Now we fix $C \in \mathcal{C}$ and $x_{1}, x_{i} \in \mathbb{M}\left(\mathbb{C}_{\infty}\right)$. Then the number of distinct $x_{j} \in \mathbb{M}\left(\mathbb{C}_{\infty}\right)$ such that $\left(x_{1}, x_{i}, x_{j}\right) \in p_{\{1, i, j\}}(C)$ is bounded from below by an increasing function in $\left|N_{C, j}\right|$ (Proposition 2.20). But at least $1 / r$ of these points $x_{j}$ must also satisfy $\left(x_{i}, x_{j}\right) \in Y_{0}^{\prime}(M)$, of which there can be at most $\psi(M)$. It follows that $\left|N_{C, i}\right|$ and 
$\left|N_{C, j}\right|$ are bounded independently of $C \in \mathcal{C}$. Restricting $\mathcal{C}$ once again, we may assume that $p_{i, j}(C)=Y_{0}^{\prime}\left(N_{0}\right)$ for all $C \in \mathcal{C}$.

Now one can show that $Y \cong Y_{0}^{\prime}\left(N_{0}\right) \times \mathbb{M}^{n-2}$, which is special.

Step 3. It remains to show that the primes $\mathfrak{p}_{1}, \ldots, \mathfrak{p}_{d-1} \subset A$ satisfying (i)(iv) above actually exist, if the constant $B>0$ is chosen sufficiently large. Let $x=\left(x_{1}, \ldots, x_{n}\right) \in S$ such that $H_{\mathrm{CM}}\left(x_{i}\right)=q^{g_{i}}\left|\mathfrak{f}_{i}\right|>B$ for all $i=1, \ldots, n$.

As before, the problem boils down to finding simultaneous solutions $t_{1}, \ldots, t_{d-1} \in$ $2 n_{c} \mathbb{N}$ to the following four inequalities:

$$
\begin{gathered}
q^{t_{1}} \geq \max (13, \operatorname{deg}(Y)), \\
C_{\varepsilon}\left(q^{g_{i}}\left|\mathfrak{f}_{i}\right|\right)^{1-\varepsilon}>[F: k] q^{2 t_{d-1}}\left(2 q^{t_{d-1}}+2\right)^{n}, \quad \text { for some } \varepsilon>0 \text { and some } 1 \leq i \leq n, \\
q^{t_{j+1}} \geq(\operatorname{deg}(Y))^{2^{j}} \prod_{m=1}^{j}\left(2 q^{t_{m}}+2\right)^{n 2^{j-m}}, \quad \text { for all } 1 \leq j \leq d-1, \\
C_{1} q^{t_{j}} / t_{j}-\left(C_{2}\left(g_{1}+\cdots+g_{n}\right)+C_{3}\right) q^{t_{j} / 2}>\log _{q}\left|\mathfrak{f}_{1} \cdots \mathfrak{f}_{n}\right|, \quad \text { for all } 1 \leq j \leq d-1 .
\end{gathered}
$$

If $B>0$ is sufficiently large, then such solutions exist. This completes the proof of Theorem 1.2 ,

\section{Application to Heegner points}

In this section we apply Theorem 1.2 to extend the main result of [2] to arbitrary global function fields of odd characteristic.

Let $E$ be an elliptic curve defined over $k$, which we recall is a global function field over $\mathbb{F}_{q}$, with $q$ odd. Suppose that the $j$-invariant of $E$ is not constant, so we say that $E$ is non-isotrivial. It follows that $j(E)$ has negative valuation at some place, and hence $E$ has potential split multiplicative reduction there. Thus, replacing $k$ by a finite extension if necessary, there exists a place of $k$ at which $E$ has split multiplicative reduction, and if we call this place $\infty$ we are in the situation of the previous sections. Now the conductor of $E$ is of the form $\mathfrak{n} \cdot \infty$, for an ideal $\mathfrak{n} \subset A$. It follows from the work of Drinfeld and others that we have a modular parametrization

$$
\pi: X_{0}(\mathfrak{n}) \longrightarrow E
$$

defined over $k$, where $X_{0}(\mathfrak{n})$ is the smooth projective model for the curve $Y_{0}(\mathfrak{n})$ defined in $\$ 2.3$. See 8 for a detailed treatment.

We fix a prime $\mathfrak{p}$ of $\mathrm{A}$ for the remainder of this section.

Lemma 4.2. There exist infinitely many quadratic imaginary extensions $K / k$ satisfying the following two conditions:

(i) Every prime $\mathfrak{q} \subset A, \mathfrak{q} \neq \mathfrak{p}$, which ramifies in $K / k$ is principal in $k$.

(ii) Every prime $\mathfrak{q} \subset A$ which divides $\mathfrak{n}$ splits in $K / k$ (Heegner hypothesis).

Proof. Denote by $k_{\mathfrak{n}}$ the ray class field of $k$ with conductor $\mathfrak{n}$. Then a prime $\mathfrak{q} \subset A$ splits completely in $k_{\mathfrak{n}}$ if and only if $\mathfrak{q}=\langle x\rangle$ with $x \equiv 1 \bmod \mathfrak{n}$. Denote by $\mathcal{Q}_{\mathfrak{n}}$ the set of primes $\mathfrak{q} \subset A$ of odd degree which split completely in $k_{\mathfrak{n}}$. By the Cebotarev Theorem [5, Prop. 5.16], this set is infinite. Now let $m \in A$ such that $\langle m\rangle$ is a product of primes in $\mathcal{Q}_{\mathfrak{n}}$ and $\operatorname{deg}(m)$ is odd. Then $k(\sqrt{m}) / k$ is a quadratic imaginary extension satisfying conditions (i) and (ii) above. 
Fix a quadratic imaginary extension $K / k$ satisfying conditions 4.2(i)-(ii) above, and let $n \in \mathbb{N}$. Denote by $\mathcal{O}_{K}$ the ring of integers of $K$, and let $\mathcal{O}_{n}=A+$ $\mathfrak{p}^{n} \mathcal{O}_{K}$, which is an order of conductor $\mathfrak{p}^{n}$ in $\mathcal{O}_{K}$. Thanks to condition 4.2(ii), there exists an ideal $\mathfrak{N}_{n} \subset \mathcal{O}_{n}$ such that $\mathcal{O}_{n} / \mathfrak{N}_{n} \cong A / \mathfrak{n}$ as $A$-modules. It follows that the pair of lattices $\left(\mathcal{O}_{n}, \mathfrak{N}_{n}^{-1}\right)$ defines a pair of Drinfeld modules with complex multiplication by $\mathcal{O}_{n}$ and linked by a cyclic $\mathfrak{n}$-isogeny. Thus the pair defines a point $x_{n} \in X_{0}(\mathfrak{n})\left(K\left[\mathfrak{p}^{n}\right]\right)$, where $K\left[\mathfrak{p}^{n}\right]$ denotes the ring class field of $\mathcal{O}_{n}$.

We let $K[\infty]:=\bigcup_{n \geq 0} K\left[\mathfrak{p}^{n}\right]$ in a chosen algebraic closure $\bar{k}$ of $k$. Then

$$
\operatorname{Gal}(K[\infty] / K) \cong G_{0} \times \mathbb{Z}_{p}^{\infty},
$$

where $\mathbb{Z}_{p}^{\infty}$ denotes the direct product of countably many copies of $\mathbb{Z}_{p}^{+}$, where $p$ is the characteristic of $k$, and $G_{0}$ is a finite abelian group; see [2, Proposition 2.1]. We denote by $H[\infty] \subset K[\infty]$ the fixed field of $G_{0}$, so $\operatorname{Gal}(K[\infty] / H[\infty])=G_{0}$ and $\operatorname{Gal}(H[\infty] / K) \cong \mathbb{Z}_{p}^{\infty}$. We write $H\left[\mathfrak{p}^{n}\right]=H[\infty] \cap K\left[\mathfrak{p}^{n}\right]$.

We now define the $n$th higher Heegner point on $E$ by

$$
y_{n}:=\operatorname{Tr}_{G_{0}}\left(\pi\left(x_{n}\right)\right)=\sum_{\sigma \in G_{0}} \pi\left(x_{n}^{\sigma}\right) \in E\left(H\left[\mathfrak{p}^{n}\right]\right) .
$$

The main result of this section is

Theorem 4.4. Let $I \subset \mathbb{N}$ be an infinite subset. In the above situation, the group generated by $\left\{y_{n} \mid n \in I\right\}$ in $E(H[\infty])$ has finite torsion and infinite rank.

Proof. Most of the work has already been done in [2, Theorem 2], combined with Theorem 1.2 of the current paper. It remains to verify the surjectivity of a new modular parametrization $\pi^{\prime}: X_{0}(\mathfrak{m n}) \rightarrow E$, which we proceed to describe.

Let $\mathfrak{p}_{1}, \ldots, \mathfrak{p}_{g}$ denote the primes $\neq \mathfrak{p}$ of $A$ which ramify in $K / k$. As we have assumed condition $4.2(\mathrm{i})$, we know that they are all principal ideals. Let $\mathfrak{m}=$ $\mathfrak{p}_{1} \cdots \mathfrak{p}_{g}$ denote their product. Choose a set $S \subset \mathrm{GL}_{2}\left(\mathbb{A}_{f}\right)$ of representatives for the double quotient $\mathrm{GL}_{2}(k) \backslash \mathrm{GL}_{2}\left(\mathbb{A}_{f}\right) / \mathcal{K}_{0}(\mathfrak{n})$. Then $S$ is also a set of representatives for $\mathrm{GL}_{2}(k) \backslash \mathrm{GL}_{2}\left(\mathbb{A}_{f}\right) / \mathcal{K}_{0}(\mathfrak{n m})$ as $\mathcal{K}_{0}(\mathfrak{n m}) \subset \mathcal{K}_{0}(\mathfrak{n})$, and $\operatorname{det}\left(\mathcal{K}_{0}(\mathfrak{n})\right)=\operatorname{det}\left(\mathcal{K}_{0}(\mathfrak{n m})\right)=$ $\hat{A}^{\times}$(see 22.3 for notation). For each $s \in S$ we write $\Gamma_{s}(\mathfrak{n})=s \mathcal{K}_{0}(\mathfrak{n}) s^{-1} \cap \mathrm{GL}_{2}(k)$ and $\Gamma_{s}(\mathfrak{n m})=s \mathcal{K}_{0}(\mathfrak{n m}) s^{-1} \cap \mathrm{GL}_{2}(k)$. Setting $\Omega^{*}=\Omega \cup \mathbb{P}^{1}(k)$, on which $\mathrm{GL}_{2}(k)$ acts in the obvious way, we get

$$
\begin{aligned}
X_{0}(\mathfrak{n})\left(\mathbb{C}_{\infty}\right)^{\text {an }} & \cong \coprod_{s \in S} \Gamma_{s}(\mathfrak{n}) \backslash \Omega^{*}, \\
X_{0}(\mathfrak{n} \mathfrak{m})\left(\mathbb{C}_{\infty}\right)^{\text {an }} & \cong \coprod_{s \in S} \Gamma_{s}(\mathfrak{n} \mathfrak{m}) \backslash \Omega^{*} .
\end{aligned}
$$

Every divisor $\mathfrak{d} \mid \mathfrak{m}$ is principal, and we write $\mathfrak{d}=\langle d\rangle$ for a chosen $d \in A$. Let $D$ denote a set of these $d$ 's as $\mathfrak{d}$ ranges through all divisors of $\mathfrak{m}$, so $|D|=2^{g}$.

We define the full degeneracy map $\delta: X_{0}(\mathfrak{n m}) \rightarrow X_{0}(\mathfrak{n})^{2^{g}}$ by its action on $\mathbb{C}_{\infty^{-}}$ valued points:

$$
\delta:[\omega] \longmapsto([d \omega])_{d \in D} \quad \text { on each } \Gamma_{0}(\mathfrak{n m}) \backslash \Omega^{*} .
$$

Next, we give an analytic description of the modular parametrization (4.1); see [8] for details. Denote by $\mathcal{T}_{\infty}$ the Brûhat-Tits tree of $\mathrm{GL}_{2}\left(k_{\infty}\right)$, and by $\underline{H}\left(\mathcal{T}_{\infty}, \mathbb{Z}\right)$ the group of $\mathbb{Z}$-valued harmonic cochains on the set of edges of $\mathcal{T}_{\infty}$. For each $s \in S$ one associates to $E$ a primitive Hecke newform $\varphi_{s} \in \underline{H}_{!}\left(\mathcal{T}_{\infty}, \mathbb{Z}\right)^{\Gamma_{s}(\mathfrak{n})}$, the latter group denoting those harmonic cochains invariant under $\Gamma_{s}(\mathfrak{n})$-action and with compact 
(= finite) support on $\Gamma_{s}(\mathfrak{n}) \backslash \mathcal{T}_{\infty}$. To $\varphi_{s}$ one associates a certain holomorphic theta function $u_{s}: \Omega \rightarrow \mathbb{C}_{\infty}^{\times}$with multiplier $c_{s}: \Gamma_{s}(\mathfrak{n}) \rightarrow \mathbb{C}_{\infty}^{\times}$; in other words, $u_{s}(\alpha \omega)=$ $c_{s}(\alpha) u_{s}(\omega)$ for all $\omega \in \Omega$ and $\alpha \in \Gamma_{s}(\mathfrak{n})$. We let $\Delta_{s}=\left\{c_{s}(\alpha) \mid \alpha \in \Gamma_{s}(\mathfrak{n})\right\}$, which is a multiplicative lattice in $\mathbb{C}_{\infty}^{\times}$. The elliptic curve $E$, which has split multiplicative reduction at $\infty$, is isomorphic to the Tate curve $\mathbb{C}_{\infty}^{\times} / \Delta_{s}$, for each $s \in S$. Finally, on each $s$-component of $Y_{0}(\mathfrak{n})$, the modular parametrization (4.1) is given explicitly on $\mathbb{C}_{\infty}$-valued points by

$$
\begin{aligned}
\Gamma_{s} \backslash \Omega & \longrightarrow \mathbb{C}_{\infty}^{\times} / \Delta_{s}, \\
{[\omega] } & \longmapsto u_{s}(\omega) \bmod \Delta_{s},
\end{aligned}
$$

and the cusps $X_{0}(\mathfrak{n}) \backslash Y_{0}(\mathfrak{n})$ map to the identity of $E$.

Now we can combine (4.1) with (4.5) to obtain a new modular parametrization of $E$ :

$$
\begin{aligned}
& \pi^{\prime}: X_{0}(\mathfrak{n m}) \stackrel{\Sigma \circ \pi \circ \delta}{\longrightarrow} E, \\
& {[\omega] \quad \prod_{d \in D} u_{s}(d \omega) \bmod \Delta_{s}, \quad \text { on each } \Gamma_{s}(\mathfrak{n} \mathfrak{m}) \backslash \Omega \text {. }}
\end{aligned}
$$

According to [2, Theorem 2] it remains for us to show that $\pi^{\prime}: \Gamma_{s}(\mathfrak{n m}) \backslash \Omega^{*} \rightarrow E$ is surjective for every $s \in S$, in other words, that $u_{s}^{\prime}(\omega):=\prod_{d \in D} u_{s}(d \omega)$ is not constant.

Denote by $\mathcal{O}_{\Omega}(\Omega)$ the ring of rigid analytic functions on $\Omega$. Then there is an exact sequence

$$
1 \longrightarrow \mathbb{C}_{\infty}^{\times} \longrightarrow \mathcal{O}_{\Omega}(\Omega)^{\times} \stackrel{r}{\longrightarrow} \underline{H}\left(\mathcal{T}_{\infty}, \mathbb{Z}\right) \longrightarrow 0,
$$

and the homomorphism $r$ satisfies $r(f \circ \alpha)=r(f) \circ \alpha$ for all $\alpha \in \mathrm{GL}_{2}(k)$. Moreover, $r\left(u_{s}\right)=\varphi_{s}$.

Now suppose that $u_{s}^{\prime}$ is constant. Then

$$
0=r\left(u_{s}^{\prime}\right)=\sum_{d \in D} \varphi_{s} \circ\left(\begin{array}{ll}
d & 0 \\
0 & 1
\end{array}\right) .
$$

Now $\varphi_{s} \neq 0$, so there exists an edge $e_{0}$ of $\mathcal{T}_{\infty}$ such that $\varphi_{s}\left(e_{0}\right) \neq 0$. Thus by (4.7) there is some $1 \neq d_{0} \in D$ such that $\varphi_{s}\left(\left(\begin{array}{cc}d_{0} & 0 \\ 0 & 1\end{array}\right) \cdot e_{0}\right) \neq 0$. Write $e_{1}=\left(\begin{array}{cc}d_{0} & 0 \\ 0 & 1\end{array}\right) \cdot e_{0}$, and we again find some $1 \neq d_{1} \in D$ such that $\varphi_{s}\left(\left(\begin{array}{cc}d_{1} & 0 \\ 0 & 1\end{array}\right) \cdot e_{1}\right) \neq 0$, and so on, giving us an infinite sequence $e_{0}, e_{1}, \ldots$, of edges of $\mathcal{T}_{\infty}$ with $\varphi_{s}\left(e_{i}\right) \neq 0$ for all $i \geq 0$. Moreover, as $\left|d_{i}\right|>1$ for all $i$, we see that the $e_{i}$ 's form a sequence of distinct edges all lying on one end of $\mathcal{T}_{\infty}$. It follows that their images in $\Gamma_{s}(\mathfrak{n}) \backslash \mathcal{T}_{\infty}$ still form an infinite sequence of edges lying on one end of $\Gamma_{s}(\mathfrak{n}) \backslash \mathcal{T}_{\infty}$. But this contradicts the fact that $\varphi_{s}$ has compact support modulo $\Gamma_{s}(\mathfrak{n})$, which completes the proof of Theorem 4.4 .

Remark 4.8. The assumption 4.2(i) should not be necessary, but the trouble begins when, if we have non-principal ramified primes other than $\mathfrak{p}$, the generalization of (4.7) mixes Hecke newforms $\varphi_{s}$ for several different $s \in S$ in a single equation.

\footnotetext{
${ }^{1}$ In 2] we mistakenly only required $\pi^{\prime}: X_{0}(\mathfrak{n m}) \rightarrow E$ to be surjective, whereas we actually need surjectivity for each irreducible component of $X_{0}(\mathfrak{n m})$.
} 


\section{ACKNOWLEDGMENTS}

Most of this paper was written at the Max-Planck-Institut für Mathematik in Bonn, Germany, and the author would like to thank the Insitut and its members for their hospitality. The author is also grateful to Gebhard Böckle for pointing out the trick used to complete the proof of Theorem 4.4.

\section{REFERENCES}

1. Y. André, "Finitude des couples d'invariants modulaires singuliers sur une courbe algébrique plane non modulaire", J. Reine Angew. Math. 505 (1998), 203-208. MR 1662256 (2000a:11090)

2. F. Breuer, "Higher Heegner points on elliptic curves over function fields", J. Number Theory 104 (2004), 315-326. MR2029509 (2005g:11092)

3. F. Breuer, "The André-Oort conjecture for products of Drinfeld modular curves", J. Reine Angew. Math. 579 (2005), 115-144. MR2124020 (2005k:11113)

4. S.J. Edixhoven, "Special points on products of modular curves", Duke Math. J. 126 (2005), no. 2, 325-348. MR 2115260

5. M. Fried and M. Jarden, "Field Arithmetic", Springer-Verlag, 1986. MR0868860 (89b:12010)

6. W. Fulton, "Intersection Theory", Springer-Verlag, 1984. MR0732620 (85k:14004)

7. E.-U. Gekeler, "Drinfeld Modular Curves", Lecture Notes in Mathematics 1231, SpringerVerlag, 1986. MR0874338 (88b:11077)

8. E.-U. Gekeler and M. Reversat, "Jacobians of Drinfeld modular curves", J. Reine Angew. Math. 476 (1996), 27-93. MR.1401696 (97f:11043)

9. D. Goss, "Basic Structures of Function Field Arithmetic", Springer-Verlag, 1996. MR1423131 (97i:11062)

10. D. Hayes, "Explicit class field theory in global function fields", in: "Studies in algebra and number theory" (G.C.Rota, ed.), Academic Press, New York, 1979. MR0535766 (81d:12011)

11. D. Hayes, "A Brief introduction to Drinfeld modules", in: The Arithmetic of Function Fields (eds. D. Goss et al.), de Gruyter, New York, Berlin, 1992. MR1196509 (93m:11050)

12. L.K. Hua, appendix to: J. Dieudonné, "On the automorphisms of the classical groups", Memoirs Amer. Math. Soc. 2 (1951), 1-95. MR0606555(82c:20079)

13. G.-J. van der Heiden, "Weil pairing and the Drinfeld modular curve", Ph.D. thesis, Rijksuniversiteit Groningen, 2003.

14. W. Lütkebohmert, "Der Satz von Remmert-Stein in der nichtarchimedischen Funktionentheorie", Math. Z. 139 (1974), 69-84. MR0352527 (50:5014)

15. J. Neukirch, "Algebraische Zahlentheorie", Springer-Verlag, 1992. MR.1697859 (2000m:11104)

16. J.-P. Serre, "Trees", Springer-Verlag, 1980. MR0607504 (82c:20083)

17. H. Stichtenoth, "Algebraic Function Fields and Codes", Springer-Verlag, 1993. MR1251961 (94k:14016)

Department of Mathematical Sciences, University of Stellenbosch, Stellenbosch, 7600, South Africa

E-mail address: fbreuer@sun.ac.za 